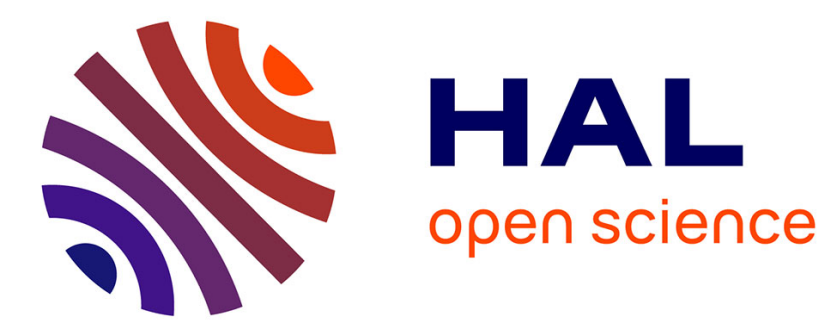

\title{
Development and Applications of Fluorogen/Light-Up RNA Aptamer Pairs for RNA Detection and More
}

\author{
Michael Ryckelynck
}

\section{To cite this version:}

Michael Ryckelynck. Development and Applications of Fluorogen/Light-Up RNA Aptamer Pairs for RNA Detection and More. RNA Tagging Methods and Protocols, pp.73-102, 2020, Methods in molecular biology, 10.1007/978-1-0716-0712-1_5 . hal-02953767

\section{HAL Id: hal-02953767 \\ https://hal.science/hal-02953767}

Submitted on 30 Sep 2020

HAL is a multi-disciplinary open access archive for the deposit and dissemination of scientific research documents, whether they are published or not. The documents may come from teaching and research institutions in France or abroad, or from public or private research centers.
L'archive ouverte pluridisciplinaire HAL, est destinée au dépôt et à la diffusion de documents scientifiques de niveau recherche, publiés ou non, émanant des établissements d'enseignement et de recherche français ou étrangers, des laboratoires publics ou privés. 


\title{
Development and applications of fluorogen/light-up RNA aptamer pairs for RNA detection and more
}

\section{Michael Ryckelynck*}

Université de Strasbourg, CNRS, Architecture et Réactivité de l'ARN, UPR 9002, F-67000 Strasbourg, France

*:m.ryckelynck@unistra.fr

\begin{abstract}
The central role of RNA in living systems made it highly desirable to have non-invasive and sensitive technologies allowing for imaging the synthesis and the location of these molecules in living cells. This need motivated the development of small pro-fluorescent molecules called 'fluorogens' that become fluorescent upon binding to genetically encodable RNAs called 'light-up aptamers'. Yet, the development of these fluorogen/light-up RNA pairs is a long and thorough process starting with the careful design of the fluorogen and pursued by the selection of a specific and efficient synthetic aptamer. This chapter summarizes the main design and the selection strategies used up to now prior to introducing the main pairs. Then, the vast application potential of these molecules for live-cell RNA imaging and other applications will be presented and discussed.
\end{abstract}

Running title: Light-up RNA aptamers

Keywords: fluorogen, aptamer, RNA, functional screening, SELEX, live-cell imaging, biosensing, engineering. 


\section{Introduction}

RNA is a main actor of cell life mainly through its central role in gene expression and its regulation. Indeed, RNA has pleiotropic functions such as being the message (messenger RNA or mRNA) to be translated into protein, the regulator of gene transcription, mRNA maturation and/or translation (e.g., non-coding regulatory RNAs) as well as being the active component of key cellular machineries (e.g., ribosomal RNA, small nuclear RNAs, and RNase P). It is therefore of prime importance to be able to monitor the expression and ideally also the location of RNAs all along the cellular lifespan. Yet, for a long time, the detection of cellular nucleic acids was restricted to the use of non-specific intercalating dyes (e.g., Hoechst [1] and cyanines [2]) and to in situ hybridization (ISH) methodologies [3], in which radioactively or fluorescently labelled oligonucleotides (DNA or RNA) are used to detect RNA molecules upon specific annealing of the probe. Whereas ISH approaches allow detecting nucleic acids with great specificity and sensitivity, they also require cells to be fixed and permeabilized, which compromises live-cell imaging and leads to a significant loss of information on the dynamics of the biological system. Other probes and nanoparticles such as molecular beacons and nanoflares have been proposed as an alternative to fluorescent ISH (FISH) but these probes face cell-entry and toxicity issues [4].

A first breakthrough in live-cell RNA imaging came with the introduction of the "RBP-FP" methods pioneered by Bertrand et al in late 90's with the so-called MS2-GFP system [5]. These approaches exploit the capacity of some RNA-binding proteins (RBP) to specifically recognize short RNA motifs herein used as RNA tags. The RNA to image is then expressed in cells in fusion (usually in the 3' untranslated region of mRNAs) with an array of RNA tags (several tens of motifs tandemly repeated). In addition, a construct coding for the RBP specific to the RNA tag and fused with a fluorescent protein (FP), such as the Green Fluorescent Protein (GFP), is also expressed in the same cell. As a consequence, upon synthesis, the target mRNA is rapidly decorated with tens of GFPs turning it into a highly fluorescent object that can be imaged with single molecule resolution [6]. Yet, whereas this technology allowed collecting highly valuable data on mRNA synthesis, addressing and distribution in the cell, it also suffers significant drawbacks linked to the large size of the tag array (up to 32 repeats of the RNA motif), especially when decorated by the RBP-FP, as well as the background coming from the constitutively expressed RBP-FP; though adding a Nuclear Localization Sequence can be used to confine unbound RBP-FP into the nucleus. These features preclude the use of this technology for imaging small, yet highly relevant, non-coding RNAs but also its application to organisms deprived of internal compartments (e.g., bacteria). Yet, these major limitations can be overcome by reducing the length of RNA tag arrays and by exchanging the bulky fluorescent RBP-FP for a smaller pro-fluorescent molecule.

Conversely to proteins, no naturally fluorescent RNA has been discovered yet, making it necessary to use a pro-fluorescent co-factor. In 2003 the group of Roger Tsien reported that the so-called MGA RNA aptamer binds specifically to malachite green and other triphenylmethane dyes and that this 
interaction activates their fluorescence [7], making these dyes fluorogenic (Figure 1). Since this pioneering work, a large variety of such dyes ('fluorogens') and RNA aptamers ('light-up aptamers') forming specific fluorogen/light-up aptamer pairs has been developed and they are still subject of a very active field of research today [8-10]. This chapter aims at reviewing and discussing the development of the main fluorogen/ light-up aptamer pairs that are currently available. In addition, some of their applications in live-cell RNA imaging as well as in biosensing and nanotechnology will be presented.

\section{Development of fluorogen/light-up RNA aptamer pairs}

As highlighted above, RNA has no intrinsic fluorescence capacity, but it can acquire fluorescence through specific binding of a fluorogen. Ideally, this fluorogen co-factor should (1) emit no fluorescence in its free state, (2) bind the RNA aptamer with high affinity (sub-micromolar or even sub-nanomolar $K_{D}$ ), (3) produce strong fluorescence upon binding, and (4) be photostable. All these properties rely on both partners as demonstrated below. As a consequence, the development of an efficient fluorogen/aptamer pair is a long and complex multiparametric process in which the fluorogen should be properly designed and the RNA aptamer subsequently identified by using a suited selection strategy. This strategy led to the identification of several tens of pairs covering all the visible spectrum and displaying ever-improved properties (Table 1).

\subsection{Design of the fluorogen}

Malachite Green (MG) was the first fluorogen found to be specifically activated by an RNA aptamer [7]. This was rather an unexpected discovery, since the aptamer was originally developed to bind MG with the idea of producing reactive radicals aimed at cleaving RNA in close proximity [11]. The true development of dedicated fluorogens (most of them are shown on Figure 2) started shortly after, leading to a first generation of molecules based on known nucleic acids-binding dyes such as Hoechst and cyanines like Thiazole Orange (TO) and Oxazole Orange (YO). These molecules are intrinsically profluorescent. Indeed, Hoechst is an environment-sensitive molecule that does not emit fluorescence in its free state, but becomes highly fluorescent when bound to DNA [9]. On their side, TO and YO have the capacity to eliminate an excitation energy via intramolecular movements in their free state. However, binding to nucleic acids hinders intramolecular movements and the excitation energy is then eliminated by fluorescence emission. To serve as specific fluorogen, the non-specific binding capacity of these dyes was strongly attenuated by introducing blocking chemical groups, leading to compounds with a potential to interact with nucleic acids but becoming fluorescent only upon binding to a specific sequence. This first set of fluorogens encompasses molecules like Hoechst derivative 1c [12,13], TO1-Biotin [14], TO3biotin [14], YO3 [15], DIR [16], DIR-pro [17] and OTB-SO3 [17] (Table 1). In addition to these repurposed dyes, bio-inspired fluorogens (also behaving as molecular rotors) were designed by mimicking fluorophores found in fluorescent proteins. In doing so, the group of Samie Jaffrey introduced DFHBI (3,5-difluoro-4-hydroxybenzylidene imidazolinone) as the first GFP-mimicking fluorogen [18] together 
with DFHBI-1T, a brighter derivative [19]. Moreover, since green fluorescence may also originate from cell auto-fluorescence, several groups later developed red-emitting FP-mimicking fluorogens [20-22]. Interestingly, protonated forms of these FP-mimicking fluorogens (e.g., DMHBI+, DMHBI-Imi and $\mathrm{DMHBO}_{+}$) represent large stoke-shift fluorogens, which are particularly attractive for Förster Resonance Energy Transfer (FRET) applications [22]. This first generation of fluorogens already covered most of the visible spectrum (Figure 2) and allowed a real breakthrough to begin in RNA imaging technologies (see below). However, these dyes suffer from a limited brightness (Table 1) and, in most of the cases, of a low photostability - the most extreme case being encountered with the DFHBI and DFHBI-1T (from now summarized as DFHBI(-1T)) fluorogens that, when associated with their specific aptamer, produce fluorescence for less than a second before getting photobleached [23,24].

The above-mentioned limitations encouraged the development of a second set of fluorogens based on organic dyes known to be both bright and photostable (e.g., rhodamines, Atto and Alexa). Yet, these molecules first needed to be converted into reversible non-emissive species (Figure 2). To do so, the molecule is usually conjugated with a quenching moiety that prevents fluorescence emission either by contact quenching, FRET or electron transfer [25]. For instance, direct addition of an aniline to an amino group of a sulforhodamine core led to ASR, a dye quenched by an electron transfer mechanism revertible upon aptamer binding [26]. Sulforhodamine B (SRB) can also be conjugated to dinitroaniline (DN) via a short polyethylene glycol (PEG) linker introduced at the level of a sulfone group, yielding a fluorogen (i.e., SR-DN) rendered non-emissive by a contact-quenching phenomenon, [27,28]. The beauty of this approach is the possibility of generating a variety of dyes with different colors. Indeed, grafting DNPEG to various dyes with xanthene-like cores (e.g., RG-DN and TMR-DN) allowed generating a rainbow of fluorogens [29] and led recently to the development of the very bright Silicon RhodamineDN fluorogen [30]. Interestingly, exploiting the internal spirocyclization (a spontaneous intramolecular cyclization event abrogating the fluorescence of the molecule but reversible by the binding to an aptamer) capacity of the Silicon Rhodamine even made the addition of the quencher moiety (i.e., SRlinker) dispensable, yet at the expense of a lower fluorescence turn-on, thus leading to a lower contrast [30]. Alternative designs in which DN was exchanged for FRET quenchers like Black Hole Quencher 1 (e.g., Cy3-BHQ1) [31] and, more recently, Cobalamin (e.g., Cbl-Cy5 and Cbl-Atto590) [32] were also described and enabled the development of multicolor imaging platforms. The use of Cobalamin $(\mathrm{Cbl})$ as a quencher is a very clever strategy as it exploits a natural compound for which several natural and specifically interacting RNA aptamer sequences were already identified in bacterial riboswitches. Fortunately, this interaction with $\mathrm{Cbl}$ is efficient enough to abrogate the quenching of the dye, making the de novo isolation of a dedicated aptamer dispensable.

\subsection{Development and properties of artificial light-up RNA aptamers}

With the exception of Cbl-binding RNAs introduced above, no other natural light-up RNA aptamer has been described yet. While the absence of such molecule in cells limits the risk of unwanted 
background fluorescence resulting from a cross activation by endogenous sequences, it also requires the development of an artificial aptamer. Over the past decade, several strategies have been proposed to isolate and optimize light-up RNA aptamers.

\subsubsection{Aptamer isolation strategies}

Traditionally, artificial aptamers are obtained using an in vitro selection technology known as SELEX (Systematic Evolution of Ligands by EXponential enrichment) introduced in the early 90's $[33,34]$. In this approach, RNA (or DNA) molecules contained in large libraries made of 1014-15 different sequences are challenged to interact with an immobilized target; here, a fluorogen (Figure 3a). Upon stringent washes, molecules displaying sufficient affinity for the target are recovered and the resulting enriched library is used to prime another round of selection. As a consequence, aptamers isolated by SELEX are likely to be specific and high affinity binders. Consistent with this assumption, most of the aptamers recognize their fluorogen with nanomolar affinity (Table 1). The best systems described so far in this regard are the TO1-Biotin/Mango [14], DMHBO+/Chili [22] and TMR-DN/SRB2 systems showing $K_{D}$ values of 3, 12 and $35 \mathrm{nM}$ respectively [29].

Yet, since during SELEX no selection pressure is applied for the capacity of the aptamer to turn on the fluorogen, isolated RNAs are not necessarily expected to be efficient light-up molecules. In agreement with this statement, it has been reported that within the final pool of DFHBI-binding aptamers, less than $1 \%$ of the molecules were actually fluorogenic [35]. Furthermore, except from SiRA (Table 1), all the SELEX-derived aptamers reported so far form complexes of sub-optimal brightness. The brightness of a fluorogen/aptamer complex results from the number of photons the fluorogen can absorb (quantified by the absorption coefficient $\varepsilon$ ) and from the number of photons re-emitted per absorbed photons (quantified by the quantum yield QY). Therefore, to be as bright as possible, a complex should have both the highest possible $\varepsilon$ value and a QY as close as possible to 1 (i.e., every absorbed photon is re-emitted). Consequently, even though DFHBI-1T/Spinach2 displays an excellent QY of 0.98 , the low $\varepsilon$ of the complex makes it just as bright as eGFP. On the contrary, complexes with much higher $\varepsilon$ such as TO1-Biotin/Mango, DIR-Pro/DIR2s and Cbl-Cy5/Riboglow are limited by their low QY (Table 1). Therefore, to be efficient, the aptamer selection pipeline should ideally include a screening step during which the capacity of each molecule to light-up the fluorogen is also taken into account.

Following the strategy originally used to identify improved variants of GFP [36], the aptamer variants contained in a SELEX-enriched pool can be further selected for light-up aptamers upon expression in bacteria grown on agar plates supplemented with fluorogen and selecting colonies that are fluorescent [26]. However, this approach allows only analyzing a few hundreds of aptamers. Significant gain in throughput can be reached by analyzing bacteria with a Fluorescence Activated Cell Sorter (FACS, Figure 3b). Indeed, combining SELEX pre-enrichment with FACS screening of aptamerexpressing bacteria allowed to identify Broccoli, a DFHBI-binding aptamer optimized for imaging RNA 
in living cells [35]. While live-cell FACS screening allows to select aptamers with lighting-up capacity directly in the cellular context, it may also face significant drawbacks given by the cell-based system such as (i) limited bacteria transformation efficiency, (ii) requirement of the fluorogen to be cell membrane-permeable (dispensable for the development of extra-cellular probes), or (iii) difficulty to apply harsh and controlled selection pressures. To overcome these limitations, aptamer libraries should ideally be screened in vitro. Such in vitro screening can be performed using Gene-linked RNA Aptamer Particle (GRAP) display [37], a technology in which aptamer-coding genes contained in a library are first individualized at the surface of beads onto which they are then clonally amplified by emulsion PCR. Upon washing, beads are emulsified in a second set of droplets into which DNA is transcribed into RNA aptamers that are then captured on the bead surface. Finally, incubating beads with a fluorogen allows to fluorescently label the beads all the better the aptamer is efficient and makes it possible to FACS-sort them (Figure 3b). Advantageously, this method enables to select for both brightness and affinity and was successfully used to isolate new MG-binding aptamers forming higher affinity or brighter complexes with the fluorogen. Such a screening would have been extremely challenging to perform with living cells given the significant toxicity of the fluorogen [38]. Recently, the methodology was further improved by making the second emulsification step (in vitro transcription step) dispensable. Indeed, in the so-called R-CAMPS technology the RNA produced from DNA coupled on the bead surface is cotranscriptionally captured at the surface of the same bead via a specific sequence [39]. The functionality of this approach was demonstrated by screening small libraries of Baby Spinach aptamer. However, the significant polydispersity of the emulsions generated during PCR and in vitro transcription steps may affect the overall efficiency of the bead-based methods. Thus, a significant gain in accuracy could be reached by performing the amplification and transcription reactions in more homogenous emulsions.

An attractive way of producing highly identical water-in-oil droplets is to use microfluidics as it allows to generate and manipulate highly monodisperse emulsions and to gain control over selection conditions. In this view, we recently introduced microfluidic-assisted In Vitro Compartmentalization ( $\mu$ IVC) as an alternative to the in vitro ultrahigh-throughput screening approach [40]. In $\mu$ IVC, genes contained in a library are individualized into small water-in-oil droplets (Figure 3c) in which they are PCR-amplified, expressed into RNA (or even protein) and analyzed for their properties (e.g., enzyme activity, capacity to light-up a fluorogen...). The droplets with the genes of interest are then sorted at rates of several millions per day. Such ultrahigh-throughput is primarily possible due to the extreme miniaturization of the reaction vessels (down to a few picoliters) and the use of microfluidic devices that allow to produce highly monodisperse emulsions and to manipulate (fusion, injection and sorting) individual droplets with electricity (avoiding the use of moving parts that would limit the throughput) [41]. We first demonstrated $\mu$ IVC efficiency in light-up aptamer development by improving the folding and fluorescence properties of the light-up aptamer Spinach, which led to the isolation of iSpinach, an aptamer optimized for in vitro applications [42]. Yet, so far, the best illustration of $\mu$ IVC efficiency was obtained with the isolation of new Mango aptamers. Indeed, the original Mango aptamer was found to 
form a high affinity complex with the fluorogen TO1-Biotin, but the complex also suffered from a low QY of 0.14, making it dimmer than half an eGFP (Table 1). Re-screening the SELEX-enriched library from which Mango was identified using a $\mu \mathrm{IVC}$ procedure enabled us to identify three new Mango variants (Mango-II, III and IV) [43]. Of these news aptamers, Mango-II displayed an improved affinity for TO1-Biotin while having unchanged lighting-up capacity, whereas Mango-III (the major sequence found at the end of process) had preserved affinity for the fluorogen while forming a complex with a nearly 4-fold higher QY, making it significantly brighter than eGFP (Table 1). Interestingly, Mango-III displays important differences with the other Mango aptamers both at the sequence and the structure level (see below). Moreover, Mango III dominated the pool at the end of the screening process, whereas it was completely missed during the previous hand screenings [14]. Taken together, the case of MangoIII demonstrates how powerful the combined use of SELEX and $\mu$ IVC can be at identifying efficient light-up RNA aptamers.

Finally, also microarrays were used for in vitro functional screening of aptamers [44-46]. Upon DNA spotting onto a surface, genes are transcribed and captured either onto a functionalized coverslip [46] or into a dedicated micro-chamber [44]. Then, the RNA is incubated with the fluorogen and the array imaged. Moreover, using a microfluidic device it became possible to keep the array under perfusion of liquid and to vary the concentration of the fluorogen (or other compounds) while collecting thermodynamic data on the system [44]. Whereas array-based technologies operate at much lower throughput than $\mu \mathrm{IVC}$ and are therefore less efficient at isolating optimal light-up aptamers from large pools, the possibility to directly associate each phenotype to the encoding genotype makes these techniques particularly well-suited for the fine functional characterization of light-up aptamers via the screening of comprehensive single (if not double) point mutant libraries.

\subsubsection{Structure-assisted characterization and optimization of light-up aptamers}

Once a light-up aptamer has been identified, the most efficient way to understand the molecular mechanism driving the recognition and the activation of the fluorogen is to solve the crystal structure of the fluorogen/aptamer complex. Moreover, such structural characterization is highly valuable to further engineer the system (e.g., to develop biosensors and supramolecular assemblies, see below). Thus, nearly half of the systems presented in table 1 have been crystalized and their structure solved. Even though each aptamer adopts an idiosyncratic folding, they all share the presence of an extended platform made of a base-triple [47], a base-quadruple [48] or even a G-quartet [49-54] that accommodates the fluorogen [53]. Fluorogens are polycyclic compounds and their optimal activation is usually obtained by holding them in planar conformation while properly confining them within a binding pocket.

On a structural point of view, the original Mango aptamer possesses one of the simplest fluorogenbinding pockets, which consists of a G-quartet surmounted by three unpaired nucleotides (two As and a U) that belong to G-quadruplex propellers (Figure 4a) [52]. As a consequence, one face of the fluorogen is still largely exposed to the solvent, explaining in part the low QY of the complex (Table 1). Moreover, 
both cycles of the TO1 fluorogen are rotated relative to each other instead of being co-planar as required to obtain maximal fluorescence emission. Mango-II shares the same overall structure except that five adenines are contributed by the G-quadruplex propellers and allow to better constrain and accommodate the fluorogen [54] without significantly modifying the environment of the dye (Figure 4b). Moreover, while held in planar conformation, the fluorogen is also slightly more exposed to the solvent. Taken together, these observations explained the increased affinity of Mango-II for TO1-Biotin (better accommodation of the fluorogen) while the brightness of the complex remained unchanged (no advantageous modification of the fluorogen environment). Mango-I and II are both able to trigger TO1Biotin fluorescence and to a lower extend that of the related, red-emitting analogue TO3-Biotin. However, guided by the crystal structure, we identified a point mutant of Mango-II (Mango-II (A22U)) endowed with a better capacity to discriminate both fluorogens [54]. Interestingly, and as expected from the significant sequence differences, Mango-III displays a rather different structural organization [55]. Indeed, whereas the RNA still possesses a G-quadruplex of which the top quartet forms the fluorogenbinding platform, the aptamer has a much more compact and robust structure in which almost every residue is involved in hydrogen bonding. Moreover, and conversely to other Mangos, the G-quartet platform of Mango-III is not surmounted by unpaired nucleobases but is rather capped by a A-U basepair (A10-U17) that sandwiches the fluorogen and is stabilized by an intercalating $U$ (U12) residue (Figure 4c). As a direct consequence of this tight accommodation of TO1-Biotin between the Gquadruplex platform and the apical A-U base-pair, the QY of TO1-Biotin/Mango-III is almost four times higher than that of other Mango aptamers without significant change in affinity (Table 1). Moreover, we found out that Mango-III almost completely lost the capacity to trigger TO3-Biotin fluorescence, making this aptamer one of the most specific aptamer known to date. As before, guided by the crystal structure, we identified 10 residues that could potentially be sub-optimal [55]. We prepared a mutant library in which these 10 positions were randomized, and we screened it for improved variants using $\mu I V C$. Excitingly, this allowed us to identify iMango-III, an aptamer forming an even brighter complex with TO1-Biotin and with preserved affinity (Table 1). The overall structure of iMango-III is identical to that of Mango-III [55,56], the main difference being the exchange of the A-U apical closing base-pair for a $U \cdot U$ pair that was identified as being responsible for the increase in brightness of the complex.

Recently, the crystal structure of the DIR2s aptamer was solved in complex with the fluorogen OTBSO3 [47]. Interestingly, the fluorogen-binding platform of DIR2s consists only of a base triple, and the fluorogen is capped by a single adenine (A40, Figure 4d). Moreover, the fluorogen establishes a specific interaction with a guanine (G39) of the platform via its sulfone moiety. Interestingly, it was proposed that the absence of G-quadruplex should render this aptamer less sensitive to the cellular G-quadruplex unfolding machinery recently discovered [57]. However, the relaxed constrain exerted on the fluorogen has also two main drawbacks, i.e. a moderate brightness (moderate QY) and a lack of specificity of the complex (the aptamer was found to bind and trigger fluorescence not only OTB-SO3 but also of DIRPro and TO1-Biotin). Yet, both limitations could be overcome by re-exploring the SELEX-enriched 
libraries using a functional screening approach like $\mu \mathrm{IVC}$. Interestingly, the fluorogen-binding platform of MGA does not consist in a G-quartet either but instead consists of a base quadruple $(\mathrm{G} \bullet \mathrm{G} \bullet \mathrm{A} \cdot \mathrm{C})$ that accommodates the fluorogen which is further capped by a $\mathrm{G} \cdot \mathrm{C}$ base-pair (G8-C28) and surrounded by several unpaired residues (Figure 4e) [48]. Despite this a priori tight accommodation, the complex is still characterized by a low QY (0.19), but recent experiments showed that this value could be significantly increased using in vitro functional screening [37].

Among the different structurally characterized light-up RNA aptamers, DFHBI-(1T)-binding Spinach aptamers (Spinach [18], Spinach2 [58], iSpinach [42]) possess the most elaborated fluorogenbinding pocket (Figure 4f). Indeed, all the solved crystal structures showed that the fluorogen-binding platform is made of a G-quartet and that DFHBI(-1T) is accommodated between this platform and a base-triple, while specific contacts are established with a side guanine (G31) [49-51]. Exploiting the crystal structure allowed to truncate Spinach to a much shorter aptamer called Baby-Spinach that preserves the properties of the parental molecule [50]. In addition to the Spinach family aptamers, it is very likely that the same DFHBI(-1T)-binding pocket is shared by Broccoli [35]. Indeed, even though no crystal structure has been solved for this aptamer, the close sequence proximity with Spinach strongly suggests that both molecules adopt the same folding [59-61]. However, despite the tight apparent accommodation of the fluorogen, all the known DFHBI(-1T)/aptamer complexes suffer from a very short fluorescence half-life (less than 1 second) [23]. This is likely due to the loose constrain applied to the imidazolinone moiety that stays free to eliminate part of the excitation energy through rapid photoisomerization.

The precursor of Broccoli was recently subjected to rounds of directed evolution and converted into Orange Broccoli and Red Broccoli, two aptamers able to light-up DFHO, a red fluorescent proteinmimicking fluorogen, at different wavelengths [21]. Comparing the sequences of the three (the original, Orange and Red) Broccolis as well as considering their putative Spinach-like DFHBI(-1T)-binding pocket allowed to identify a single nucleotide responsible for the Broccoli spectral tuning [60]. While this feature makes Broccoli an interesting precursor for the development of multicolor tags, this aptamer family is still limited by the poor photostability and the limited brightness of the complex they form with their fluorogens [21]. However, significant gain in photostability of the FP-mimicking fluorogens was recently reached with the isolation of Corn, a DFHO-binding aptamer protecting its fluorogen from the rapid photo-isomerization encountered with Spinach and Broccoli families [21]. Interestingly, this impressive gain in photostability was not attributable to the fluorogen itself (which does not display such a stability when bound to Broccoli aptamers) but rather to the way the aptamer accommodates it. Indeed, the crystal structure of DFHO/Corn revealed that the RNA is organized around a G-quadruplex and that the DFHO is actually accommodated between the apical quartet of two monomers forming a complex with a fluorogen:aptamer stoichiometry of 1:2. (Figure 4g) [62]. As a consequence, the DFHO is maintained in an emissive planar conformation by being sandwiched between both G-quartets while 
five unpaired adenines further restrict possible movement. Altogether, these elements prevent photoisomerization to take place and confer to DFHO/Corn a very high photostability.

\section{Imaging and sensing applications using light-up RNA aptamers}

As highlighted in the introduction of this chapter, one of the main motivations in developing fluorogens and light-up RNA aptamers was the possibility foreseen to directly monitor RNA synthesis, as well as the movement and location of RNA molecules within living cells, while overcoming limitations of RBP-FPs. The strong toxicity of Malachite Green for yeast and mammalian cells [38] largely prevented its use as a fluorogen for live-cell RNA imaging. On the contrary, all the other aboveintroduced fluorogens were found to be non-toxic, deprived of non-specific interaction with cell components and most of them were further found to be cell membrane permeable. Therefore, most of these systems are well-suited for live-cell imaging applications. Moreover, since nucleic acids are molecules highly amenable to engineering, these aptamers were rapidly converted into a variety of sensors with a wide application spectrum both in live cells and in vitro.

\subsection{General considerations before starting live-cell applications}

So far, DFHBI $(-1 \mathrm{~T})$ has been the most broadly used fluorogen, as it is commercially available from several companies and is able to enter various cell types such as, for example, bacteria [18], yeast $[63,64]$, algae [65] and mammalian cells [18,58,35]. However, other fluorogens like DFHO and TO1Biotin are also known to be cell-permeable and are now commercialized (by Lucerna and Applied Biological Material, respectively), which will further ease their wide use in the near future. Because of the short half-life of short RNAs in cells and because of limited folding capacity, fluorescence can hardly be observed upon expressing Spinach or Broccoli family aptamers as free RNA molecules (Figure 5a). Instead, aptamer RNAs should be transcribed in fusion to a tRNA [66] or an F30 scaffold [67] that assist in the proper folding and providing resistance to RNase degradation. An elegant alternative called Tornado was recently proposed in which the aptamer is flanked by a pair of ribozymes that undergo self-cleavage just after RNA synthesis leaving 5' and 3' extremities that are joined together by a cellular ligase, thus releasing a circular RNA (racRNA) endowed with an extremely long half-life [68]. To visualize these stabilized light-up RNA aptamers, they are usually expressed from strong promoters (i.e., T7 RNA polymerase promoter in prokaryotes and U6 polymerase III promoter in eukaryotes) to demonstrate both proper aptamer functionality in the cellular environment as well as the capacity of the fluorogen to cross the plasma membrane [31,18,58,27,35,28,21,43,32,15,29,30]. Moreover, proper expression and integrity of the aptamer can also be assessed by extracting and analyzing total RNA by gel electrophoresis. Staining the gel with a fluorogen (DFHBI or TO1-Biotin) then allows to specifically detect RNAs labeled with the light-up aptamer [67,69]. Yet, to be significant, the fluorogen/aptamer pair should also enable imaging less abundant but more biologically relevant RNAs.

\subsection{Live-cell RNA imaging using light-up aptamers inserted into the target RNA}


An RNA of interest (ROI) can be imaged by expressing it in fusion with the light-up aptamer (Figure 5b). This strategy was initially applied to abundant eukaryotic small non-coding RNAs transcribed by RNA polymerase III (pol. III). Indeed, labeling 5S rRNA and 7SK RNA with Spinach2 allowed visualizing them at the expected location (i.e., diffuse in the cytoplasm and in nuclear speckles, respectively) [58]. Moreover, using a construct in which 60 CGG repeats (the hallmark of some neurodegenerative diseases) were fused to Spinach2 allowed to recapitulate the aggregation process seen during the disease and to devise a drug screening strategy. Other small pol. III transcripts (i.e. $5 \mathrm{~S}$ rRNA, U6 RNA, a box C/D scaRNA or a tRNA) were also successfully detected at the expected location upon labeling with Mango-II [43] or Corn [21]. Extending this imaging strategy to less abundant pol. II transcripts (mRNAs and microRNAs) turned to be more complex because of the only moderate brightness and photostability of these first set of probes (see above). Indeed, it was possible to detect and track STL1 mRNA fused to a single copy of Spinach aptamer in yeast using a sophisticated microscopy and image analysis pipeline [63]. However, whereas this work demonstrated the great potential of light-up RNA aptamers, the complexity of the approach prevented its wide use. Significant gain in signal was obtained by using an array of up to 64 tandemly repeats of Spinach introduced into the 3' untranslated region of an ROI without affecting its life cycle [70]. Using brighter systems like TMR-DN/SRB2 allowed detecting CFP mRNA labeled with only 15 repeats of the aptamer in mammalian and GFP mRNA labeled with only 6 repeats in bacteria [29]. The number of aptamer repeats was even further recently decreased by labelling $\beta$-actin mRNA with only 4 copies of the Riboglow aptamer while still being able to observe RNA relocation in stress granules with a signal quality outperforming the MS2-GFP method [32]. Recently, direct labelling of GFP mRNA by only 5 copies of the very bright SiRA aptamer allowed to detect RNA synthesis in bacteria and to gain in imaging accuracy using STED super-resolution imaging [30].

Recently, it has been reported that the direct insertion of the probe may lead to folding interference between the labeled mRNA and the light-up aptamer, as in the case of eGFP mRNA labelled with SRB2 aptamer [71]. Yet, such an adverse effect can be avoided by inserting a Dicer cleavage site between the mRNA and the aptamer so that, upon synthesis, Dicer physically separates both molecules and thereby restores the aptamer folding capacity. However, while still informative on the synthesis of the ROI, this approach compromises the chances to track the ROI in the cell. Another way of avoiding folding interference while reducing the required genetics is to use probes acting in trans.

\subsection{Live-cell RNA imaging using light-up aptamers acting in trans}

Beside the direct insertion of a light-up aptamer into the sequence of an ROI, the labeling can also be performed in trans (Figure 5c-f). A first engineering strategy consists in transiently destabilizing a key structural element (e.g., a stem close to the fluorogen-binding site) of the aptamer by shortening and/or mutating it, while appending sequences complementary to the ROI near the destabilized stem (Figure 5c). In its free state, the RNA probe is unable to interact with the fluorogen and cannot form a 
fluorescent complex. However, upon interaction with the ROI, the light-up domain of the probe recovers its "active" folding together with its capacity to bind its fluorogen, resulting in the fluorescent labeling of the ROI. Such approach was successfully applied to endogenous mRNA visualization using engineered versions of the BHQ-1 binding aptamer [72] and of Spinach [73]. Recently, improved designs of Broccoli-derived probes with optimized sequences have been proposed and were shown to display superior performances to detect mRNA in vitro [61,74]. Yet, these probes still need to be validated in live cells. Similar design was also exploited for the development of a probe called PANDAN aimed at detecting microRNA (miR), though this probe was not evaluated in living cells either [75].

Besides stem stabilization, aptamer activation can also take place through more important structural remodeling involving strand-displacements (Figure 5d). For instance, in FASTmiR technology, an engineered version of Spinach is trapped in an inactive form and the successful binding of a target miR to a sensing domain induces, both in vitro and in live cells, several strand displacements eventually leading to the release of an active Spinach domain [76]. Substantial gain in sensitivity was later achieved by engineering a domain of the SRB2 aptamer into a molecular beacon [77]. In this construct, the successful interaction between the sensing loop of the probe and the target miR leads to structural rearrangements activating SRB2, which in turn can bind and trigger the bright SR-DN fluorogen. More recently, a new concept in which the probe is made of an L-enantiomer of Mango-III supplied from outside the cell has been introduced [78]. L-enantiomers are naturally resistant to nuclease, making these probes extremely long-lasting in challenging media (e.g., extracellular space). Furthermore, coupling the probe to a cholesterol molecule and using a clever LNA-based blocker allowed cell-entry of the probe and its activation by cellular miR (natural D-enantiomer).

Finally, a light-up aptamer can be converted into a probe acting in trans by splitting it and appending each half to a sequence complementary to the ROI (Figure 5e). Therefore, a functional aptamer is reconstituted only in the presence of the target RNA that acts as a scaffold driving aptamer assembly. Split aptamer-based methodologies were shown to offer a very high specificity [79-81]. The recent application of this concept in live cells allowed mRNA to be detected from genetically-encoded Spinach and Broccoli-derived probes with a decent sensitivity (minimum detectable concentration of 50-100 $\mathrm{nM}$ ) in living cells [82]. Substantial gain in sensitivity was further obtained by combining the use of a split Broccoli aptamer and a Catalytic Hairpin Assemblies amplification circuit (a molecular amplification circuit derived from RNA nanotechnology). The resulting CHARGE technology (Figure 5f) allows detecting the presence of as few as $2.5 \mathrm{nM}$ of target RNAs in live cells in a digital manner (i.e., informs on presence or absence of the target) [83]. Last but not least, split aptamers can also be expressed in cells and used to detect RNA-RNA interactions [84].

\subsection{In vitro detection of RNA}

The key implication of miRs in various diseases and disorders has stimulated the development of sensitive in vitro detection technologies for diagnostic applications. In this view, light-up RNA aptamers 
offer the great advantage of allowing to design cheap, label-free, and sensitive assays. Following the same concepts as for live-cell RNA imaging, the first generation of in vitro RNA sensors exploited transiently destabilized aptamers (e.g., PANDAN technology [75]) and split aptamer [81] strategies. However, the micromolar to high nanomolar sensitivity of these technologies was insufficient to detect low abundant RNAs suggesting that an amplification step may be necessary. Several RNA-based molecular amplification circuits, including CHARGE, have been developed for in vitro applications $[85,86,83]$ and could be used to increase detection sensitivity. However, an elegant alternative consisting in using the amplification capacity of the in vitro transcription reaction has recently been proposed and revealed to be extremely efficient for the sensitive detection of nucleic acids $[87,88]$ and other classes of targets (see below). Briefly, the target miR interacts with two DNA molecules (one containing the T7 RNA polymerase promoter and the second containing the sequence coding for Spinach aptamer) and drives their specific ligation to form a full-length transcription template. Then, each template is transcribed into a large number of Spinach aptamers that become fluorescent in the presence of DFHBI(1T). Such amplification-based detections allow the specific detection of miR with a low femtomolar limit of detection. In the latest format of these technologies, this limit was pushed down to the attomolar range $(5 \mathrm{aMol})$ by replacing the ligation step by a primer extension coupled with a strand displacement amplification (SDA), adding a second amplification step to the process [89].

Being able to detect RNA in vitro allows also to set-up new screening and analytical pipelines in which the in vitro transcription process can be monitored either alone [90] or in tandem with in vitro translation [91-93]. This ability to independently monitor RNA and protein (e.g., using fluorescent protein) synthesis permits to finely tune gene expression rates for applications in synthetic biology, for instance. Light-up RNA aptamers can also be used as reporters to aid in the development of catalytic RNAs endowed with self-cleaving [94] or RNA modifying activities [95].

Finally, light-up RNA aptamers also offer the great opportunity to assist and validate the design of supramolecular assemblies in RNA nanotechnology. RNA nanotechnology aims at designing programmable molecular circuits (such as the Catalytic Hairpin Assemblies introduced above) and supramolecular assemblies to achieve complex functions (e.g., computation using logic gates, channeling of catalysts on functionalized surfaces, drug delivery...) in vitro or in living cells by genetically encoding the system. For instance, light-up aptamers can be used as output signal of a molecular circuit aiming at amplifying a signal $[85,96]$ or analyzing several inputs using logic gates as complex as half-adders [97]. Incorporating a monolithic light-up aptamer into supramolecular assemblies is also frequently used to assess that RNA elements grafted onto the assembly preserve their function as well as to track these nanoobjects [98,99]. Last but not least, split versions of MGA [100103], Spinach $[102,104]$ or Broccoli $[105,106]$ revealed to be extremely useful to validate the proper assembly of multistrand RNA nanoparticles forming a variety of tiles [105,102,103] but also more elaborated shapes like cubes [100] and rings [104], just to name a few examples. 


\subsection{Detection of other biological molecules and ions}

Beyond RNA detection, light-up RNA aptamers also found a wide range of applications allowing to specifically detect and quantify several other types of target. For instance, by inserting Spinach2 into sgRNAs, it is possible to precisely localize specific loci on genomic DNA using CRISPR-display technology [107]. In a different way, fusing Spinach to the PP7 RNA sequence enables to bring the aptamer in close proximity to a PP7-mCherry fusion protein and, this way, to characterize RNA/protein interaction (e.g., affinity measurement) by monitoring FRET (Förster resonance emission transfer) between DFHBI/Spinach and mCherry fluorophores [108]. Specific protein/RNA interactions can also be established by fusing the light-up RNA to another aptamer that specifically targets a protein. This concept was applied to monitor EGFR (epidermal growth factor receptor) internalization by labelling the receptor with an EGFR-binding aptamer fused to the DIR2s aptamer [17]. As in the case described above for RNA detection, proteins can also be detected by using a bipartite probe made of a transiently destabilized light-up aptamer domain fused to a second aptamer binding specifically to the protein of interest (Figure 5g). As before, the specific interaction between the protein and the sensing aptamer stabilizes the overall structure of the probe and restores the fluorogen-binding capacity of the light-up aptamer moiety. Such allosteric probes were successfully derived from Spinach and applied to the detection of a variety of proteins synthesized in E. coli [109]. Whereas such a direct labelling is particularly well suited to monitor protein synthesis in living cells, it does not offer sufficient sensitivity to detect low abundant protein targets as potentially required for diagnostic purposes. Yet, combining conventional immunoassays like the Proximity Ligation Assay (PLA) and ELISA with the Spinach transcription-based amplification strategy introduced above allowed to devise ultrasensitive assays able to detect proteins present at picomolar [110] and attomolar [111] concentrations. Interestingly, applying the same concept to the detection of whole bacteria allowed to devise an assay able to specifically identify the presence of as few as 77 Staphylococcus aureus per $\mathrm{mL}$ of food samples [112]. Light-up aptamers can also be used to monitor the activity of enzymes, such as Dicer [113], telomerase [114] or RNA modifying enzymes [115]. These fluorogenic assays are compatible with high-throughput screening and are therefore suited for drug discovery applications, for example.

Light-up RNA aptamers also served as building blocks in the design of metabolite biosensors permitting to monitor the accumulation of a target metabolite in real time and in a non-invasive manner. Such genetically-encoded biosensors were developed, for instance, to detect SAM [116], FMN [117], TPP, cyclic AMP [118], as well as various types of cyclic dinucleotides [119-125]. In general, these sensors are based on the transient destabilization strategy (Figure 5h) similar to that described above for RNA and protein detection. These sensors are usually developed by using a trial-and-error approach, but optimal biosensors can also be identified by employing high-throughput screening approaches such as the $\mu \mathrm{IVC}$-based procedure we recently described [126]. Alternative design strategies have been proposed using natural riboswitches and circularly permutated molecules. However, since these sensors and their development are out of the scope of this chapter, the reader is redirected to some excellent 
recent reviews on the topic [127-129]. While for a long time the use of these sensors in mammalian cells was challenged by the short half-life of small RNAs in these cells, the recent development of the Tornado technology allows to express these sensors as highly stable circular RNAs [68].

Exploiting RNA nanotechnology also led to the designing of a construct comprising Spinach and Mango aptamers positioned in such a way that FRET can occur between the fluorogens (respectively DFHBI-1T and YO3) bound to each aptamer. Moreover, inserting a metabolite-sensing aptamers into the construct allows to couple the FRET signal to a metabolite sensing event [15]. Interestingly, metabolite biosensors can also be used as extra-cellular probes in screening experiments aiming at identifying microbes with improved capacity to synthesize and secrete a target metabolite [130].

Last but least, light-up aptamers can easily be converted into ions sensors. The two most prominent examples are silver and lead sensors. Indeed, simply converting a Watson-Crick base-pair of Broccoli into a $\mathrm{C} \cdot \mathrm{C}$ mismatches allowed to transiently abrogate the fluorogen-binding capacity of the aptamer. However, in the presence of silver ions, a C-Ag+- $\mathrm{C}$ metallo base-pair forms and restores aptamer capacity to bind and trigger DFHBI-1T fluorescence [131]. Expressing this sensor in living bacteria permitted to titrate the amount of silver that can actually enter and accumulate into the cells. The second example is lead detection. Indeed, it was found that the G-quadruplex structure contained in Spinach can strongly bind and get stabilized by $\mathrm{Pb}_{2}+$ ions, making Spinach an excellent sensor able to specifically detect as few as $6 \mathrm{nM} \mathrm{Pb}_{2+}$, a concentration far below the maximum permissible concentration $(72 \mathrm{nM})$ [132]. Moreover, it was recently found that a 2'-fluorinated version of the sensor may offer even higher performance by protecting the sensor from RNase action and by enhancing the association with $\mathrm{Pb}_{2+}$ ions [133]. Interestingly, exploiting this $\mathrm{Pb}_{2}+$ binding capacity of Spinach enabled the development of a fluorescence-free liquid crystal sensor able to detect $\mathrm{Pb}_{2}$ ions with similar selectivity and sensitivity [134], demonstrating how properties of light-up aptamers can even be used without exploiting their fluorescence capacity. In the same line, the very high affinity of Mango for TO1-Biotin can be exploited in experiments aiming to specifically isolate target RNAs and bound molecules by affinity capture [135].

\section{Conclusions}

Since the discovery that an RNA molecule may have the capacity to light-up a fluorogen [7], many groups have started to develop highly efficient, bright, photostable and cell-permeable fluorogens together with their specific aptamers. To this end, strong progresses were made in the chemistry of the fluorogen but also in the development of new technologies and methodologies for high-throughput functional screening of mutant gene libraries as well as for the stabilization of RNA probes both inside (e.g., Tornado [68]) and outside (e.g., use of 2'-fluorinated ribose [133] and L-enantiomers [78]) the cell.

While the main motivation in developing new efficient fluorogen/light-up aptamer pairs was mainly driven by the possibility of imaging RNA in living cells with high sensitivity, one can see that the application scope of these probes rapidly diversified toward the sensitive sensing of a variety of 
molecules (DNA, protein, metabolites and ions) both in live-cell cells and in vitro. Indeed, the development and the commercialization of ultrasensitive detection kits may have a profound impact on various fields such as healthcare and environment survey. Light-up aptamers may also play a role in drug discovery by allowing to set-up high-throughput screening pipelines as well as by assisting the development of RNA nanoobjects that could act, for instance, as drug delivery cargos.

As a conclusion, it is likely that even more efficient fluorogen/light-up aptamer pairs will be developed over the coming years and that additional types of molecules beyond RNA will be used for the development of light-up aptamers. Indeed, two DNA-based light-up aptamers have been described $[12,20]$ which may provide great advantages in term of cost and backbone stability. Moreover, one can expect that the application spectrum of light-up aptamers will continue to grow far beyond RNA detection and that first analysis kits and devices using these molecules may appear on the market in a near future. Therefore, the story of light-up aptamers is just beginning.

\section{References}

1. Jin R, Breslauer KJ (1988) Characterization of the minor groove environment in a drug-DNA complex: bisbenzimide bound to the poly[d(AT)].poly[d(AT)]duplex. Proc Natl Acad Sci U S A 85 (23):8939-8942

2. Armitage BA (2008) Cyanine dye-nucleic acid interactions. In: Strekowski L (ed) Heterocyclic Polymethine Dyes: Synthesis, Properties and Applications. Springer Berlin Heidelberg, Berlin, Heidelberg, pp 11-29. doi:10.1007/7081_2007_109

3. Tyagi S (2009) Imaging intracellular RNA distribution and dynamics in living cells. Nat Methods 6 (5):331338. doi:10.1038/nmeth. 1321

4. Wang Z, Liu W, Fan C, Chen N (2019) Visualizing mRNA in live mammalian cells. Methods. doi:10.1016/j.ymeth.2019.03.008

5. Bertrand E, Chartrand P, Schaefer M, Shenoy SM, Singer RH, Long RM (1998) Localization of ASH1 mRNA particles in living yeast. Mol Cell 2 (4):437-445

6. Buxbaum AR, Haimovich G, Singer RH (2015) In the right place at the right time: visualizing and understanding mRNA localization. Nat Rev Mol Cell Biol 16 (2):95-109. doi:10.1038/nrm3918

7. Babendure JR, Adams SR, Tsien RY (2003) Aptamers switch on fluorescence of triphenylmethane dyes. J Am Chem Soc 125 (48):14716-14717. doi:10.1021/ja037994o

8. Ouellet J (2016) RNA Fluorescence with light-up aptamers. Front Chem 4:29. doi:10.3389/fchem.2016.00029

9. Bouhedda F, Autour A, Ryckelynck M (2018) Light-up RNA aptamers and their cognate fluorogens: from their development to their applications. Int J Mol Sci 19 (1):44. doi:10.3390/ijms19010044

10. Neubacher S, Hennig S (2019) RNA structure and cellular applications of fluorescent light-up aptamers. Angew Chem Int Ed Engl 58 (5):1266-1279. doi:10.1002/anie.201806482

11. Grate D, Wilson C (1999) Laser-mediated, site-specific inactivation of RNA transcripts. Proc Natl Acad Sci U S A 96 (11):6131-6136

12. Sando S, Narita A, Aoyama Y (2007) Light-up Hoechst-DNA aptamer pair: generation of an aptamer-selective fluorophore from a conventional DNA-staining dye. Chembiochem 8 (15):1795-1803. doi:10.1002/cbic. 200700325 
13. Sando S, Narita A, Hayami M, Aoyama Y (2008) Transcription monitoring using fused RNA with a dyebinding light-up aptamer as a tag: a blue fluorescent RNA. Chem Commun (Camb) 44 (33):3858-3860. doi:10.1039/b808449a

14. Dolgosheina EV, Jeng SC, Panchapakesan SS, Cojocaru R, Chen PS, Wilson PD, Hawkins N, Wiggins PA, Unrau PJ (2014) RNA mango aptamer-fluorophore: a bright, high-affinity complex for RNA labeling and tracking. ACS Chem Biol 9 (10):2412-2420. doi:10.1021/cb500499x

15. Jepsen MDE, Sparvath SM, Nielsen TB, Langvad AH, Grossi G, Gothelf KV, Andersen ES (2018) Development of a genetically encodable FRET system using fluorescent RNA aptamers. Nat Commun 9 (1):18. doi:10.1038/s41467-017-02435-x

16. Constantin TP, Silva GL, Robertson KL, Hamilton TP, Fague K, Waggoner AS, Armitage BA (2008) Synthesis of new fluorogenic cyanine dyes and incorporation into RNA fluoromodules. Org Lett 10 (8):1561-1564. doi:10.1021/ol702920e

17. Tan X, Constantin TP, Sloane KL, Waggoner AS, Bruchez MP, Armitage BA (2017) Fluoromodules consisting of a promiscuous RNA aptamer and red or blue fluorogenic cyanine dyes: selection, characterization, and bioimaging. J Am Chem Soc 139 (26):9001-9009. doi:10.1021/jacs.7b04211

18. Paige JS, Wu KY, Jaffrey SR (2011) RNA mimics of green fluorescent protein. Science 333 (6042):642-646. doi:10.1126/science.1207339

19. Song W, Strack RL, Svensen N, Jaffrey SR (2014) Plug-and-Play Fluorophores Extend the Spectral Properties of Spinach. J Am Chem Soc 136 (4):1198-1201. doi:10.1021/ja410819x

20. Feng G, Luo C, Yi H, Yuan L, Lin B, Luo X, Hu X, Wang H, Lei C, Nie Z, Yao S (2017) DNA mimics of red fluorescent proteins (RFP) based on G-quadruplex-confined synthetic RFP chromophores. Nucleic Acids Res 45 (18):10380-10392. doi:10.1093/nar/gkx803

21. Song W, Filonov GS, Kim H, Hirsch M, Li X, Moon JD, Jaffrey SR (2017) Imaging RNA polymerase III transcription using a photostable RNA-fluorophore complex. Nat Chem Biol 13 (11):1187-1194. doi:10.1038/nchembio. 2477

22. Steinmetzger C, Palanisamy N, Gore KR, Hobartner C (2019) A multicolor large Stokes shift fluorogenactivating RNA aptamer with cationic chromophores. Chemistry 25 (8):1931-1935. doi:10.1002/chem.201805882

23. Han KY, Leslie BJ, Fei J, Zhang J, Ha T (2013) Understanding the photophysics of the spinach-DFHBI RNA aptamer-fluorogen complex to improve live-cell RNA imaging. J Am Chem Soc 135 (50):19033-19038. doi:10.1021/ja411060p

24. You M, Jaffrey SR (2015) Structure and mechanism of RNA mimics of green fluorescent protein. Annu Rev Biophys 44:187-206. doi:10.1146/annurev-biophys-060414-033954

25. Klymchenko AS (2017) Solvatochromic and fluorogenic dyes as environment-sensitive probes: design and biological applications. Acc Chem Res 50 (2):366-375. doi:10.1021/acs.accounts.6b00517

26. Lee J, Lee KH, Jeon J, Dragulescu-Andrasi A, Xiao F, Rao J (2010) Combining SELEX screening and rational design to develop light-up fluorophore-RNA aptamer pairs for RNA tagging. ACS Chem Biol 5 (11):1065-1074. doi:10.1021/cb1001894

27. Sunbul M, Jaschke A (2013) Contact-mediated quenching for RNA imaging in bacteria with a fluorophorebinding aptamer. Angew Chem Int Ed Engl 52 (50):13401-13404. doi:10.1002/anie.201306622

28. Arora A, Sunbul M, Jaschke A (2015) Dual-colour imaging of RNAs using quencher- and fluorophore-binding aptamers. Nucleic Acids Res 43 (21):e144. doi:10.1093/nar/gkv718

29. Sunbul M, Jaschke A (2018) SRB-2: a promiscuous rainbow aptamer for live-cell RNA imaging. Nucleic Acids Res 46 (18):e110. doi:10.1093/nar/gky543 
30. Wirth R, Gao P, Nienhaus GU, Sunbul M, Jaschke A (2019) SiRA: a silicon rhodamine-binding aptamer for live-cell super-resolution RNA imaging. J Am Chem Soc 141 (18):7562-7571. doi:10.1021/jacs.9b02697

31. Murata A, Sato S, Kawazoe Y, Uesugi M (2011) Small-molecule fluorescent probes for specific RNA targets. Chem Commun (Camb) 47 (16):4712-4714. doi:10.1039/c1cc10393h

32. Braselmann E, Wierzba AJ, Polaski JT, Chrominski M, Holmes ZE, Hung ST, Batan D, Wheeler JR, Parker R, Jimenez R, Gryko D, Batey RT, Palmer AE (2018) A multicolor riboswitch-based platform for imaging of RNA in live mammalian cells. Nat Chem Biol 14 (10):964-971. doi:10.1038/s41589-018-0103-7

33. Ellington AD, Szostak JW (1990) In vitro selection of RNA molecules that bind specific ligands. Nature 346 (6287):818-822. doi:10.1038/346818a0

34. Tuerk C, Gold L (1990) Systematic evolution of ligands by exponential enrichment: RNA ligands to bacteriophage T4 DNA polymerase. Science 249 (4968):505-510. doi:10.1126/science.2200121

35. Filonov GS, Moon JD, Svensen N, Jaffrey SR (2014) Broccoli: rapid selection of an RNA mimic of green fluorescent protein by fluorescence-based selection and directed evolution. J Am Chem Soc 136 (46):1629916308. doi:10.1021/ja508478x

36. Heim R, Cubitt AB, Tsien RY (1995) Improved green fluorescence. Nature 373 (6516):663-664. doi:10.1038/373663b0

37. Gotrik M, Sekhon G, Saurabh S, Nakamoto M, Eisenstein M, Soh HT (2018) Direct selection of fluorescenceenhancing RNA aptamers. J Am Chem Soc 140 (10):3583-3591. doi:10.1021/jacs.7b10724

38. Kraus GA, Jeon I, Nilsen-Hamilton M, Awad AM, Banerjee J, Parvin B (2008) Fluorinated analogs of malachite green: synthesis and toxicity. Molecules 13 (4):986-994. doi:10.3390/molecules 13040986

39. Endoh T, Ohyama T, Sugimoto N (2019) RNA-capturing microsphere particles (R-CAMPs) for optimization of functional aptamers. Small 15 (26):e1805062. doi:10.1002/smll.201805062

40. Ryckelynck M, Baudrey S, Rick C, Marin A, Coldren F, Westhof E, Griffiths AD (2015) Using droplet-based microfluidics to improve the catalytic properties of RNA under multiple-turnover conditions. RNA 21 (3):458469. doi:10.1261/rna.048033.114

41. Autour A, Ryckelynck M (2017) Ultrahigh-throughput improvement and discovery of enzymes using dropletbased microfluidic screening. Micromachines 8 (4):128. doi:10.3390/mi8040128

42. Autour A, Westhof E, Ryckelynck M (2016) iSpinach: a fluorogenic RNA aptamer optimized for in vitro applications. Nucleic Acids Res 44 (6):2491-2500. doi:10.1093/nar/gkw083

43. Autour A, S CYJ, A DC, Abdolahzadeh A, Galli A, Panchapakesan SSS, Rueda D, Ryckelynck M, Unrau PJ (2018) Fluorogenic RNA Mango aptamers for imaging small non-coding RNAs in mammalian cells. Nat Commun 9 (1):656. doi:10.1038/s41467-018-02993-8

44. Ketterer S, Fuchs D, Weber W, Meier M (2015) Systematic reconstruction of binding and stability landscapes of the fluorogenic aptamer spinach. Nucleic Acids Res. doi:10.1093/nar/gkv944

45. Ketterer S, Gladis L, Kozica A, Meier M (2016) Engineering and characterization of fluorogenic glycine riboswitches. Nucleic Acids Res 44 (12):5983-5992. doi:10.1093/nar/gkw465

46. Henderson CA, Rail CA, Butt LE, Vincent HA, Callaghan AJ (2019) Generation of small molecule-binding RNA arrays and their application to fluorogen-binding RNA aptamers. Methods. doi:10.1016/j.ymeth.2019.04.021

47. Shelke SA, Shao Y, Laski A, Koirala D, Weissman BP, Fuller JR, Tan X, Constantin TP, Waggoner AS, Bruchez MP, Armitage BA, Piccirilli JA (2018) Structural basis for activation of fluorogenic dyes by an RNA aptamer lacking a G-quadruplex motif. Nat Commun 9 (1):4542. doi:10.1038/s41467-018-06942-3 
48. Baugh C, Grate D, Wilson C (2000) 2.8 A crystal structure of the malachite green aptamer. J Mol Biol 301 (1):117-128. doi:10.1006/jmbi.2000.3951

49. Huang H, Suslov NB, Li NS, Shelke SA, Evans ME, Koldobskaya Y, Rice PA, Piccirilli JA (2014) A Gquadruplex-containing RNA activates fluorescence in a GFP-like fluorophore. Nat Chem Biol 10 (8):686-691. doi:10.1038/nchembio.1561

50. Warner KD, Chen MC, Song W, Strack RL, Thorn A, Jaffrey SR, Ferre-D'Amare AR (2014) Structural basis for activity of highly efficient RNA mimics of green fluorescent protein. Nat Struct Mol Biol 21 (8):658-663. doi:10.1038/nsmb.2865

51. Fernandez-Millan P, Autour A, Ennifar E, Westhof E, Ryckelynck M (2017) Crystal structure and fluorescence properties of the iSpinach aptamer in complex with DFHBI. RNA 23 (12):1788-1795. doi:10.1261/rna.063008.117

52. Trachman RJ, 3rd, Demeshkina NA, Lau MWL, Panchapakesan SSS, Jeng SCY, Unrau PJ, Ferre-D'Amare AR (2017) Structural basis for high-affinity fluorophore binding and activation by RNA Mango. Nat Chem Biol 13 (7):807-813. doi:10.1038/nchembio.2392

53. Trachman RJ, 3rd, Truong L, Ferre-D'Amare AR (2017) Structural principles of fluorescent RNA aptamers. Trends Pharmacol Sci 38 (10):928-939. doi:10.1016/j.tips.2017.06.007

54. Trachman R, Abdolahzadeh A, Andreoni A, Cojocaru R, Knutson JR, Ryckelynck M, Unrau PJ, FerreD'Amare A (2018) Crystal structures of the Mango-II RNA aptamer reveal heterogeneous fluorophore binding and guide engineering of variants with improved selectivity and brightness. Biochemistry. doi:10.1021/acs.biochem.8b00399

55. Trachman RJ, 3rd, Autour A, Jeng SCY, Abdolahzadeh A, Andreoni A, Cojocaru R, Garipov R, Dolgosheina EV, Knutson JR, Ryckelynck M, Unrau PJ, Ferre-D'Amare AR (2019) Structure and functional reselection of the Mango-III fluorogenic RNA aptamer. Nat Chem Biol 15 (5):472-479. doi:10.1038/s41589-019-0267-9

56. Trachman RJ, 3rd, Stagno JR, Conrad C, Jones CP, Fischer P, Meents A, Wang YX, Ferre-D'Amare AR (2019) Co-crystal structure of the iMango-III fluorescent RNA aptamer using an X-ray free-electron laser. Acta Crystallogr F Struct Biol Commun 75 (Pt 8):547-551. doi:10.1107/S2053230X19010136

57. Guo JU, Bartel DP (2016) RNA G-quadruplexes are globally unfolded in eukaryotic cells and depleted in bacteria. Science 353 (6306). doi:10.1126/science.aaf5371

58. Strack RL, Disney MD, Jaffrey SR (2013) A superfolding Spinach2 reveals the dynamic nature of trinucleotide repeat-containing RNA. Nat Methods 10 (12):1219-1224. doi:10.1038/nmeth.2701

59. Ageely EA, Kartje ZJ, Rohilla KJ, Barkau CL, Gagnon KT (2016) Quadruplex-flanking stem structures modulate the stability and metal ion preferences of RNA mimics of GFP. ACS Chem Biol 11 (9):2398-2406. doi:10.1021/acschembio.6b00047

60. Filonov GS, Song W, Jaffrey SR (2019) Spectral tuning by a single nucleotide controls the fluorescence properties of a fluorogenic aptamer. Biochemistry 58 (12):1560-1564. doi:10.1021/acs.biochem.9b00048

61. Furuhata Y, Kobayashi M, Maruyama R, Sato Y, Makino K, Michiue T, Yui H, Nishizawa S, Yoshimoto K (2019) Programmable RNA detection with a fluorescent RNA aptamer using optimized three-way junction formation. RNA 25 (5):590-599. doi:10.1261/rna.069062.118

62. Warner KD, Sjekloca L, Song W, Filonov GS, Jaffrey SR, Ferre-D'Amare AR (2017) A homodimer interface without base pairs in an RNA mimic of red fluorescent protein. Nat Chem Biol 13 (11):1195-1201. doi:10.1038/nchembio. 2475

63. Guet D, Burns LT, Maji S, Boulanger J, Hersen P, Wente SR, Salamero J, Dargemont C (2015) Combining Spinach-tagged RNA and gene localization to image gene expression in live yeast. Nat Commun 6:8882. doi:10.1038/ncomms9882 
64. Zinskie JA, Roig M, Janetopoulos C, Myers KA, Bruist MF (2018) Live-cell imaging of small nucleolar RNA tagged with the broccoli aptamer in yeast. FEMS Yeast Res 18 (8). doi:10.1093/femsyr/foy093

65. Guzman-Zapata D, Dominguez-Anaya Y, Macedo-Osorio KS, Tovar-Aguilar A, Castrejon-Flores JL, DuranFigueroa NV, Badillo-Corona JA (2017) mRNA imaging in the chloroplast of Chlamydomonas reinhardtii using the light-up aptamer Spinach. J Biotechnol 251:186-188. doi:10.1016/j.jbiotec.2017.03.028

66. Ponchon L, Dardel F (2007) Recombinant RNA technology: the tRNA scaffold. Nat Methods 4 (7):571-576. doi:10.1038/nmeth1058

67. Filonov GS, Kam CW, Song W, Jaffrey SR (2015) In-gel imaging of RNA processing using broccoli reveals optimal aptamer expression strategies. Chem Biol 22 (5):649-660. doi:10.1016/j.chembiol.2015.04.018

68. Litke JL, Jaffrey SR (2019) Highly efficient expression of circular RNA aptamers in cells using autocatalytic transcripts. Nat Biotechnol 37 (6):667-675. doi:10.1038/s41587-019-0090-6

69. Yaseen IM, Ang QR, Unrau PJ (2019) Fluorescent Visualization of Mango-tagged RNA in Polyacrylamide Gels via a Poststaining Method. J Vis Exp (148). doi:10.3791/59112

70. Zhang J, Fei J, Leslie BJ, Han KY, Kuhlman TE, Ha T (2015) Tandem Spinach Array for mRNA Imaging in Living Bacterial Cells. Sci Rep 5:17295. doi:10.1038/srep17295

71. Ying ZM, Yuan YY, Tu B, Tang LJ, Yu RQ, Jiang JH (2019) A single promoter system co-expressing RNA sensor with fluorescent proteins for quantitative mRNA imaging in living tumor cells. Chem Sci 10 (18):48284833. doi:10.1039/c9sc00458k

72. Sato S, Watanabe M, Katsuda Y, Murata A, Wang DO, Uesugi M (2015) Live-cell imaging of endogenous mRNAs with a small molecule. Angew Chem Int Ed Engl 54 (6):1855-1858. doi:10.1002/anie.201410339

73. Ong WQ, Citron YR, Sekine S, Huang B (2017) Live cell imaging of endogenous mRNA using RNA-based fluorescence "turn-on" probe. ACS Chem Biol 12 (1):200-205. doi:10.1021/acschembio.6b00586

74. Soni R, Sharma D, Krishna AM, Sathiri J, Sharma A (2019) A highly efficient Baby Spinach-based minimal modified sensor (BSMS) for nucleic acid analysis. Org Biomol Chem 17 (30):7222-7227. doi:10.1039/c9ob01414d

75. Aw SS, Tang MX, Teo YN, Cohen SM (2016) A conformation-induced fluorescence method for microRNA detection. Nucleic Acids Res 44 (10):e92. doi:10.1093/nar/gkw108

76. Huang K, Doyle F, Wurz ZE, Tenenbaum SA, Hammond RK, Caplan JL, Meyers BC (2017) FASTmiR: an RNA-based sensor for in vitro quantification and live-cell localization of small RNAs. Nucleic Acids Res. doi:10.1093/nar/gkx504

77. Ying ZM, Wu Z, Tu B, Tan W, Jiang JH (2017) Genetically encoded fluorescent RNA sensor for ratiometric imaging of microRNA in living tumor cells. J Am Chem Soc 139 (29):9779-9782. doi:10.1021/jacs.7b04527

78. Zhong W, Sczepanski JT (2019) A mirror image fluorogenic aptamer sensor for live-cell imaging of microRNAs. ACS Sens 4 (3):566-570. doi:10.1021/acssensors.9b00252

79. Kolpashchikov DM (2005) Binary malachite green aptamer for fluorescent detection of nucleic acids. J Am Chem Soc 127 (36):12442-12443. doi:10.1021/ja0529788

80. Kikuchi N, Kolpashchikov DM (2016) Split spinach aptamer for highly selective recognition of DNA and RNA at ambient temperatures. Chembiochem 17 (17):1589-1592. doi:10.1002/cbic.201600323

81. Kikuchi N, Kolpashchikov DM (2017) A universal split spinach aptamer (USSA) for nucleic acid analysis and DNA computation. Chem Commun (Camb) 53 (36):4977-4980. doi:10.1039/c7cc01540b 
82. Wang Z, Luo Y, Xie X, Hu X, Song H, Zhao Y, Shi J, Wang L, Glinsky G, Chen N, Lal R, Fan C (2018) In situ spatial complementation of aptamer-mediated recognition enables live-cell imaging of native RNA transcripts in real time. Angew Chem Int Ed Engl 57 (4):972-976. doi:10.1002/anie.201707795

83. Karunanayake Mudiyanselage A, Yu Q, Leon-Duque MA, Zhao B, Wu R, You M (2018) Genetically encoded catalytic hairpin assembly for sensitive RNA imaging in live cells. J Am Chem Soc 140 (28):8739-8745. doi:10.1021/jacs.8b03956

84. Alam KK, Tawiah KD, Lichte MF, Porciani D, Burke DH (2017) A fluorescent split aptamer for visualizing RNA-RNA assembly in vivo. ACS Synth Biol. doi:10.1021/acssynbio.7b00059

85. Bhadra S, Ellington AD (2014) Design and application of cotranscriptional non-enzymatic RNA circuits and signal transducers. Nucleic Acids Res 42 (7):e58. doi:10.1093/nar/gku074

86. Akter F, Yokobayashi Y (2015) RNA signal amplifier circuit with integrated fluorescence output. ACS Synth Biol 4 (5):655-658. doi:10.1021/sb500314r

87. Tang X, Deng R, Sun Y, Ren X, Zhou M, Li J (2018) Amplified tandem spinach-based aptamer transcription enables low background miRNA detection. Anal Chem 90 (16):10001-10008. doi:10.1021/acs.analchem.8b02471

88. Ying ZM, Tu B, Liu L, Tang H, Tang LJ, Jiang JH (2018) Spinach-based fluorescent light-up biosensors for multiplexed and label-free detection of microRNAs. Chem Commun (Camb) 54 (24):3010-3013. doi:10.1039/c8cc00123e

89. Zhou M, Teng X, Li Y, Deng R, Li J (2019) Cascade transcription amplification of RNA aptamer for ultrasensitive microRNA detection. Anal Chem 91 (8):5295-5302. doi:10.1021/acs.analchem.9b00124

90. Hofer K, Langejurgen LV, Jaschke A (2013) Universal aptamer-based real-time monitoring of enzymatic RNA synthesis. J Am Chem Soc 135 (37):13692-13694. doi:10.1021/ja407142f

91. Pothoulakis G, Ceroni F, Reeve B, Ellis T (2013) The Spinach RNA aptamer as a characterization tool for synthetic biology. ACS Synth Biol. doi:10.1021/sb400089c

92. Chizzolini F, Forlin M, Cecchi D, Mansy SS (2014) Gene position more strongly influences cell-free protein expression from operons than T7 transcriptional promoter strength. ACS Synth Biol 3 (6):363-371. doi:10.1021/sb4000977

93. Chizzolini F, Forlin M, Yeh Martin N, Berloffa G, Cecchi D, Mansy SS (2017) Cell-free translation is more variable than transcription. ACS Synth Biol 6 (4):638-647. doi:10.1021/acssynbio.6b00250

94. Auslander S, Fuchs D, Hurlemann S, Auslander D, Fussenegger M (2016) Engineering a ribozyme cleavageinduced split fluorescent aptamer complementation assay. Nucleic Acids Res 44 (10):e94. doi:10.1093/nar/gkw117

95. Poudyal RR, Benslimane M, Lokugamage MP, Callaway MK, Staller S, Burke DH (2017) Selective inactivation of functional RNAs by ribozyme-catalyzed covalent modification. ACS Synth Biol 6 (3):528-534. doi:10.1021/acssynbio.6b00222

96. Bhadra S, Ellington AD (2014) A Spinach molecular beacon triggered by strand displacement. RNA 20 (8):1183-1194. doi:10.1261/rna.045047.114

97. Goldsworthy V, LaForce G, Abels S, Khisamutdinov EF (2018) Fluorogenic RNA aptamers: a nano-platform for fabrication of simple and combinatorial logic gates. Nanomaterials (Basel) 8 (12). doi:10.3390/nano8120984

98. Shu D, Shu Y, Haque F, Abdelmawla S, Guo P (2011) Thermodynamically stable RNA three-way junction for constructing multifunctional nanoparticles for delivery of therapeutics. Nat Nanotechnol 6 (10):658-667. doi:10.1038/nnano.2011.105 
99. Shu D, Khisamutdinov EF, Zhang L, Guo P (2014) Programmable folding of fusion RNA in vivo and in vitro driven by pRNA 3WJ motif of Phi29 DNA packaging motor. Nucleic Acids Res 42 (2):e10. doi:10.1093/nar/gkt885

100. Afonin KA, Bindewald E, Yaghoubian AJ, Voss N, Jacovetty E, Shapiro BA, Jaeger L (2010) In vitro assembly of cubic RNA-based scaffolds designed in silico. Nat Nanotechnol 5 (9):676-682. doi:10.1038/nnano.2010.160

101. Afonin KA, Viard M, Martins AN, Lockett SJ, Maciag AE, Freed EO, Heldman E, Jaeger L, Blumenthal R, Shapiro BA (2013) Activation of different split functionalities on re-association of RNA-DNA hybrids. Nat Nanotechnol 8 (4):296-304. doi:10.1038/nnano.2013.44

102. Chopra A, Sagredo S, Grossi G, Andersen ES, Simmel FC (2019) Out-of-plane aptamer functionalization of RNA three-helix tiles. Nanomaterials (Basel) 9 (4). doi:10.3390/nano9040507

103. Afonin KA, Danilov EO, Novikova IV, Leontis NB (2008) TokenRNA: a new type of sequence-specific, label-free fluorescent biosensor for folded RNA molecules. Chembiochem 9 (12):1902-1905. doi:10.1002/cbic. 200800183

104. O'Hara JM, Marashi D, Morton S, Jaeger L, Grabow WW (2019) Optimization of the split-Spinach aptamer for monitoring nanoparticle assembly involving multiple contiguous RNAs. Nanomaterials (Basel) 9 (3). doi:10.3390/nano9030378

105. Torelli E, Kozyra JW, Gu JY, Stimming U, Piantanida L, Voitchovsky K, Krasnogor N (2018) Isothermal folding of a light-up bio-orthogonal RNA origami nanoribbon. Sci Rep 8 (1):6989. doi:10.1038/s41598-01825270-6

106. Chandler M, Lyalina T, Halman J, Rackley L, Lee L, Dang D, Ke W, Sajja S, Woods S, Acharya S, Baumgarten E, Christopher J, Elshalia E, Hrebien G, Kublank K, Saleh S, Stallings B, Tafere M, Striplin C, Afonin KA (2018) Broccoli fluorets: split aptamers as a user-friendly fluorescent toolkit for dynamic RNA nanotechnology. Molecules 23 (12). doi:10.3390/molecules23123178

107. Shechner DM, Hacisuleyman E, Younger ST, Rinn JL (2015) Multiplexable, locus-specific targeting of long RNAs with CRISPR-Display. Nat Methods 12 (7):664-670. doi:10.1038/nmeth.3433

108. Roszyk L, Kollenda S, Hennig S (2017) Using a specific RNA-protein interaction to quench the fluorescent RNA Spinach. ACS Chem Biol 12 (12):2958-2964. doi:10.1021/acschembio.7b00332

109. Song W, Strack RL, Jaffrey SR (2013) Imaging bacterial protein expression using genetically encoded RNA sensors. Nat Methods 10 (9):873-875. doi:10.1038/nmeth.2568

110. Ying ZM, Xiao HY, Tang H, Yu RQ, Jiang JH (2018) Light-up RNA aptamer enabled label-free protein detection via a proximity induced transcription assay. Chem Commun (Camb) 54 (64):8877-8880. doi:10.1039/c8cc04498h

111. Sim J, Byun JY, Shin YB (2019) Transcription immunoassay: light-up RNA aptamer-based immunoassay using in vitro transcription. Chem Commun (Camb) 55 (25):3618-3621. doi:10.1039/c9cc00514e

112. Sheng L, Lu Y, Deng S, Liao X, Zhang K, Ding T, Gao H, Liu D, Deng R, Li J (2019) A transcription aptasensor: amplified, label-free and culture-independent detection of foodborne pathogens via light-up RNA aptamers. Chem Commun (Camb). doi:10.1039/c9cc05036a

113. Rogers TA, Andrews GE, Jaeger L, Grabow WW (2015) Fluorescent monitoring of RNA assembly and processing using the split-spinach aptamer. ACS Synth Biol 4 (2):162-166. doi:10.1021/sb5000725

114. Zhou Y, Shen S, Lau C, Lu J (2019) A conformational switch-based fluorescent biosensor for homogeneous detection of telomerase activity. Talanta 199:21-26. doi:10.1016/j.talanta.2019.02.018

115. Svensen N, Jaffrey SR (2016) Fluorescent RNA aptamers as a tool to study RNA-modifying enzymes. Cell Chem Biol 23 (3):415-425. doi:10.1016/j.chembiol.2015.11.018 
116. Paige JS, Nguyen-Duc T, Song W, Jaffrey SR (2012) Fluorescence imaging of cellular metabolites with RNA. Science 335 (6073):1194. doi:10.1126/science.1218298

117. Stojanovic MN, Kolpashchikov DM (2004) Modular aptameric sensors. J Am Chem Soc 126 (30):92669270. doi:10.1021/ja032013t

118. Sharma S, Zaveri A, Visweswariah SS, Krishnan Y (2014) A fluorescent nucleic acid nanodevice quantitatively images elevated cyclic adenosine monophosphate in membrane-bound compartments. Small 10 (21):4276-4280. doi:10.1002/smll.201400833

119. Nakayama S, Luo Y, Zhou J, Dayie TK, Sintim HO (2012) Nanomolar fluorescent detection of c-di-GMP using a modular aptamer strategy. Chem Commun (Camb) 48 (72):9059-9061. doi:10.1039/c2cc34379g

120. Kellenberger CA, Wilson SC, Sales-Lee J, Hammond MC (2013) RNA-based fluorescent biosensors for live cell imaging of second messengers cyclic di-GMP and cyclic AMP-GMP. J Am Chem Soc 135 (13):4906-4909. doi:10.1021/ja311960g

121. Kellenberger CA, Chen C, Whiteley AT, Portnoy DA, Hammond MC (2015) RNA-based fluorescent biosensors for live cell imaging of second messenger cyclic di-AMP. J Am Chem Soc 137 (20):6432-6435. doi:10.1021/jacs.5b00275

122. Kellenberger CA, Wilson SC, Hickey SF, Gonzalez TL, Su Y, Hallberg ZF, Brewer TF, Iavarone AT, Carlson HK, Hsieh YF, Hammond MC (2015) GEMM-I riboswitches from Geobacter sense the bacterial second messenger cyclic AMP-GMP. Proc Natl Acad Sci U S A 112 (17):5383-5388. doi:10.1073/pnas.1419328112

123. Hallberg ZF, Wang XC, Wright TA, Nan B, Ad O, Yeo J, Hammond MC (2016) Hybrid promiscuous (Hypr) GGDEF enzymes produce cyclic AMP-GMP (3', 3'-cGAMP). Proc Natl Acad Sci U S A 113 (7):1790-1795. doi:10.1073/pnas.1515287113

124. Inuzuka S, Matsumura S, Ikawa Y (2016) Optimization of RNA-based c-di-GMP fluorescent sensors through tuning their structural modules. J Biosci Bioeng 122 (2):183-187. doi:10.1016/j.jbiosc.2016.01.011

125. Wang C, Sinn M, Stifel J, Heiler AC, Sommershof A, Hartig JS (2017) Synthesis of all possible canonical (3'-5'-linked) cyclic dinucleotides and evaluation of riboswitch interactions and immune-stimulatory effects. J Am Chem Soc 139 (45):16154-16160. doi:10.1021/jacs.7b06141

126. Autour A, Bouhedda F, Cubi R, Ryckelynck M (2019) Optimization of fluorogenic RNA-based biosensors using droplet-based microfluidic ultrahigh-throughput screening. Methods 161:46-53. doi:10.1016/j.ymeth.2019.03.015

127. Hallberg ZF, Su Y, Kitto RZ, Hammond MC (2017) Engineering and In Vivo Applications of Riboswitches. Annu Rev Biochem 86:515-539. doi:10.1146/annurev-biochem-060815-014628

128. Karunanayake Mudiyanselage A, Wu R, Leon-Duque MA, Ren K, You M (2019) "Second-generation" fluorogenic RNA-based sensors. Methods 161:24-34. doi:10.1016/j.ymeth.2019.01.008

129. Sun Z, Nguyen T, McAuliffe K, You M (2019) Intracellular imaging with genetically encoded RNA-based molecular sensors. Nanomaterials (Basel) 9 (2). doi:10.3390/nano9020233

130. Abatemarco J, Sarhan MF, Wagner JM, Lin JL, Liu L, Hassouneh W, Yuan SF, Alper HS, Abate AR (2017) RNA-aptamers-in-droplets (RAPID) high-throughput screening for secretory phenotypes. Nat Commun 8 (1):332. doi:10.1038/s41467-017-00425-7

131. Yu Q, Shi J, Mudiyanselage A, Wu R, Zhao B, Zhou M, You M (2019) Genetically encoded RNA-based sensors for intracellular imaging of silver ions. Chem Commun (Camb) 55 (5):707-710. doi:10.1039/c8cc08796b

132. DasGupta S, Shelke SA, Li NS, Piccirilli JA (2015) Spinach RNA aptamer detects lead(II) with high selectivity. Chem Commun (Camb) 51 (43):9034-9037. doi:10.1039/c5cc01526j 
133. Savage JC, Shinde P, Bachinger HP, Davare MA, Shinde U (2019) A ribose modification of Spinach aptamer accelerates lead(ii) cation association in vitro. Chem Commun (Camb) 55 (42):5882-5885. doi: $10.1039 / \mathrm{c} 9 \mathrm{cc} 01697 \mathrm{j}$

134. Verma I, Devi M, Sharma D, Nandi R, Pal SK (2019) Liquid crystal based detection of Pb(II) ions using Spinach RNA as recognition probe. Langmuir 35 (24):7816-7823. doi:10.1021/acs.langmuir.8b04018

135. Panchapakesan SSS, Ferguson ML, Hayden EJ, Chen X, Hoskins AA, Unrau PJ (2017) Ribonucleoprotein purification and characterization using RNA Mango. RNA 23 (10):1592-1599. doi:10.1261/rna.062166.117 
Table 1. Main fluorogen/RNA light-up aptamers pairs and their properties.

\begin{tabular}{|c|c|c|c|c|c|c|c|c|c|}
\hline Fluorogen & Light-up aptamer & $\begin{array}{c}\text { Aptamer selection } \\
\text { strategy }\end{array}$ & $\begin{array}{c}K_{D} \\
(\mathrm{nM})\end{array}$ & Ex./Em. (nm) & $\begin{array}{l}\text { Abs. Coef. } \\
(\varepsilon)\end{array}$ & Q.Y. & Brightness & Rel. Brightness & Reference \\
\hline \multicolumn{2}{|c|}{ eGFP } & natural molecule & $\overline{1}$ & $490 / 508$ & 39,200 & 0.68 & 26.60 & 1.00 & {$[36]$} \\
\hline OTB-SO3 & DiR2s & SELEX & 662 & $380 / 421$ & 73,000 & 0.51 & 37.23 & 1.40 & [17] \\
\hline Hoescht-1c & Apt II-mini3-4 & SELEX & 35 & $345 / 470$ & n.a & 0.26 & n.a & n.a. & {$[12,13]$} \\
\hline DFHBI & Spinach & SELEX & 540 & $469 / 501$ & 24,300 & 0.72 & 17.50 & 0.65 & {$[18]$} \\
\hline DFHBI & iSpinach & $\mu \mathrm{IVC}$ & 920 & $442 / 503$ & n.a. & n.a. & n.a. & n.a. & [42] \\
\hline DFHBI-1T & Spinach2 & SELEX/design & 560 & $482 / 505$ & 31,000 & 0.94 & 29.10 & 1.10 & [19] \\
\hline DFHBI-1T & Broccoli & SELEX/FACS & 360 & $472 / 507$ & 29,600 & 0.94 & 27.80 & 1.04 & [35] \\
\hline TO1-Biotin & iMangoIII & $\mu \mathrm{IVC}$ & 4 & $506 / 527$ & 77,500 & 0.64 & 49.6 & 1.86 & {$[55]$} \\
\hline RG-DN & DNB & SELEX & 4480 & $507 / 534$ & 37,350 & 0.32 & 11.90 & 0.44 & [28] \\
\hline TO1-Biotin & Mango & SELEX & 3 & $510 / 535$ & 77,500 & 0.14 & 10.85 & 0.40 & [14] \\
\hline TO1-Biotin & MangoII & $\mu \mathrm{IVC}$ & 1 & $510 / 535$ & 77,500 & 0.21 & 16.28 & 0.61 & [43] \\
\hline TO1-Biotin & MangoIII & $\mu \mathrm{IVC}$ & 5 & $510 / 534$ & 77,500 & 0.55 & 42.63 & 1.6 & [43] \\
\hline DMHBI+ & Chili & SELEX/design & 63 & $413 / 542$ & n.a. & 0.40 & n.a. & n.a. & [22] \\
\hline DMHBI-Imi & Chili & SELEX/design & 71 & $463 / 545,594$ & n.a. & 0.08 & n.a. & n.a. & [22] \\
\hline DFHO & Corn & SELEX & 70 & $505 / 545$ & 29,000 & 0.25 & 7.25 & 0.27 & [21] \\
\hline DFHO & Orange Broccoli & SELEX & 230 & $513 / 562$ & 34,000 & 0.28 & 9.52 & 0.36 & [21] \\
\hline CY3-BHQ1 & BHQ apt (A1) & SELEX & n.a. & $520 / 565$ & n.a. & n.a. & n.a. & n.a. & [31] \\
\hline DFHO & Red-Broccoli & SELEX & 206 & $518 / 582$ & 35,000 & 0.34 & 11.90 & 0.44 & [21] \\
\hline TMR-DN & SRB2 & SELEX & 35 & $564 / 587$ & 90,500 & 0.33 & 29.87 & 1.12 & [29] \\
\hline SR-DN & DNB & SELEX & 800 & $572 / 591$ & 50,250 & 0.98 & 49.24 & 1.85 & [28] \\
\hline DMHBO+ & Chili & SELEX/design & 12 & $456 / 592$ & n.a. & 0.10 & n.a. & n.a. & [22] \\
\hline SR-DN & SRB2 & SELEX & 1340 & $579 / 596$ & 85,200 & 0.65 & 55.38 & 2.08 & [29] \\
\hline Cbl-Atto590 & Riboglow & natural molecule & $3-34$ & $594 / 624$ & 120,000 & 0.31 & 37.2 & 1.40 & [32] \\
\hline DIR & DIR apt & SELEX & 86 & $600 / 646$ & 134,000 & 0.26 & 34.80 & 1.30 & {$[16]$} \\
\hline Mal. Green & MGA & SELEX & 117 & $630 / 650$ & 150,000 & 0.19 & 28.00 & 1.05 & {$[11,7]$} \\
\hline DIR-pro & DIR2s-Apt & SELEX & 252 & $600 / 658$ & 164,000 & 0.33 & 54.12 & 2.00 & {$[17]$} \\
\hline TO3-Biotin & Mango & SELEX & $6-8$ & $637 / 658$ & 9,300 & n.a. & n.a. & n.a. & [14] \\
\hline SiR-DN & SiRA & SELEX & 1000 & $650 / 662$ & n.a. & n.a. & n.a. & n.a. & [30] \\
\hline Cbl-Cy5 & Riboglow & natural molecule & n.a. & $646 / 662$ & 271,000 & 0.26 & 70.46 & 2.65 & {$[32]$} \\
\hline Patent Blue & SRB apt & SELEX & 23 & n.a./665 & n.a. & 0.034 & n.a. & n.a. & {$[7]$} \\
\hline SiR-linker & SiRA & SELEX & 430 & $649 / 666$ & 86,000 & 0.98 & 84.28 & 3.16 & [30] \\
\hline
\end{tabular}




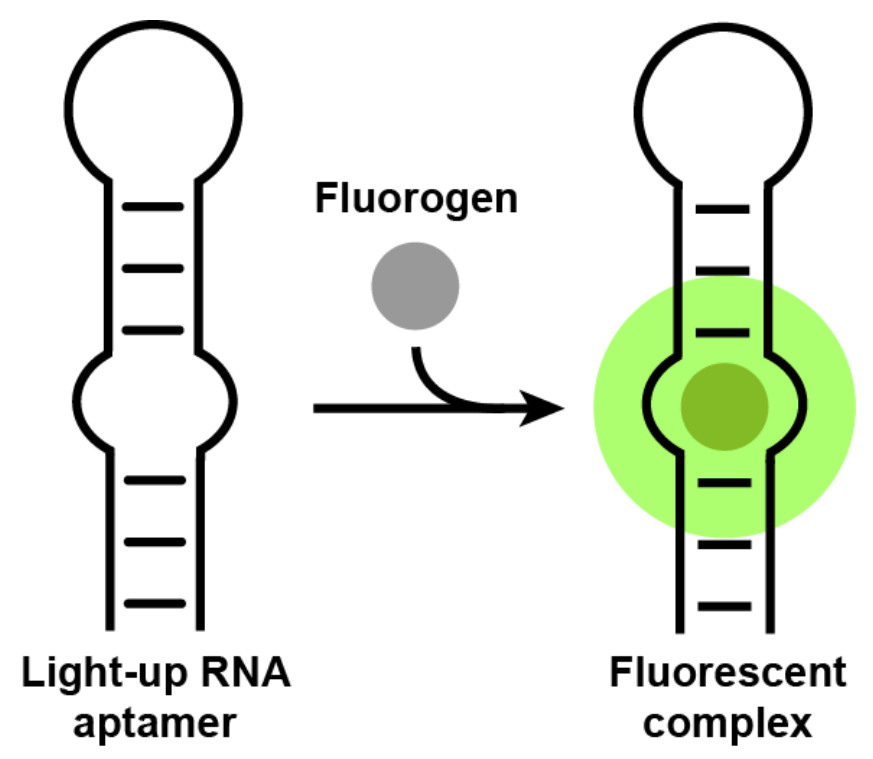

Figure 1. Formation of a fluorogen/light-up aptamer fluorescent complex. In its free state, the fluorogen (gray) is poorly emissive. However, upon the proper accommodation into the fluorogenbinding site of a light-up RNA aptamer (black) the fluorescence capacity of the molecule is restored (green) and a fluorescent complex is formed. 


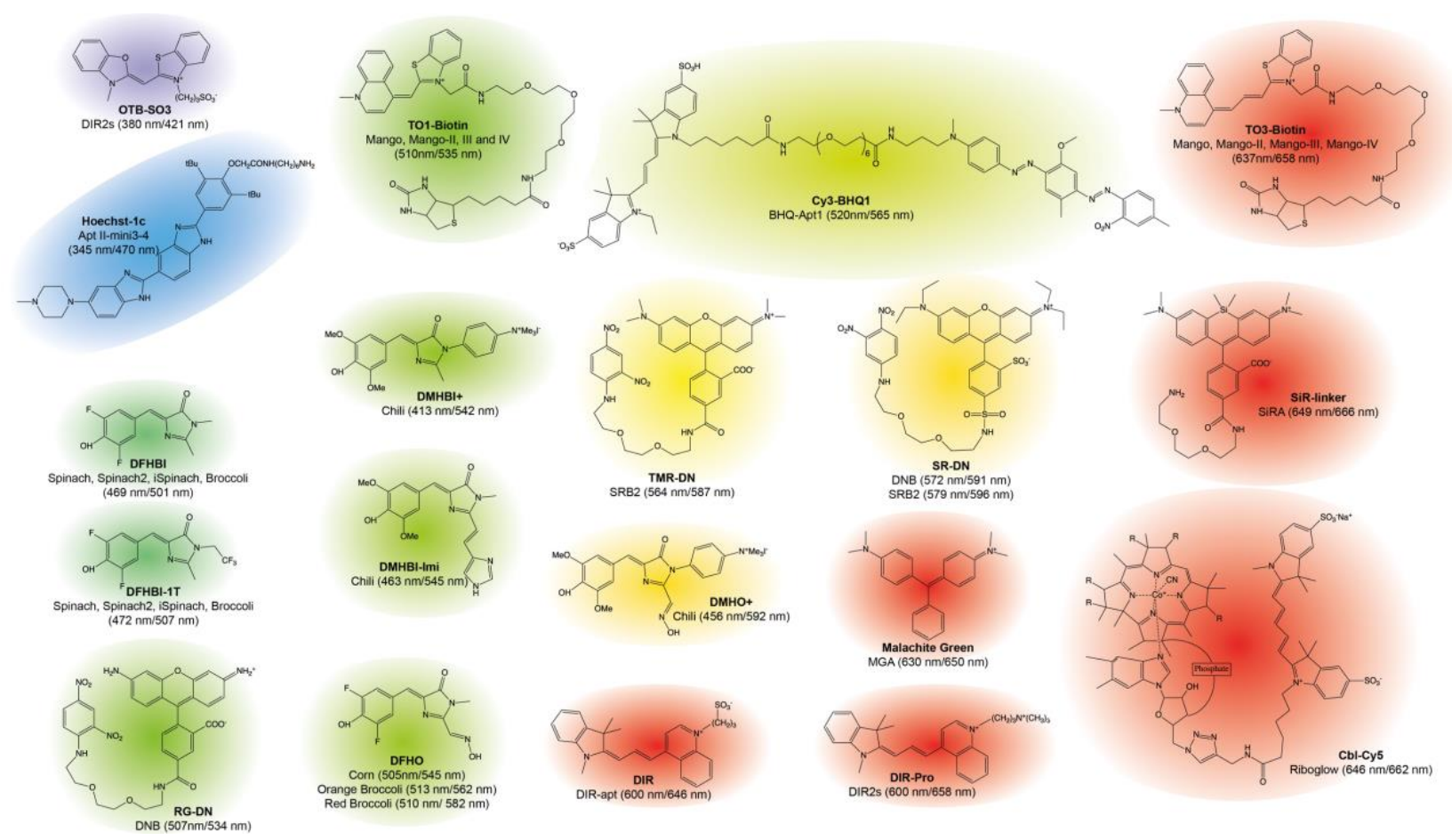

Figure 2. Structure of the main fluorogens activated by light-up RNA aptamers. The structure of each fluorogen is shown together with the name of its activating aptamer(s) and the excitation/emission wavelengths are given in parentheses. The fluorogens are ordered by their emission wavelength according to table 1 . 
a

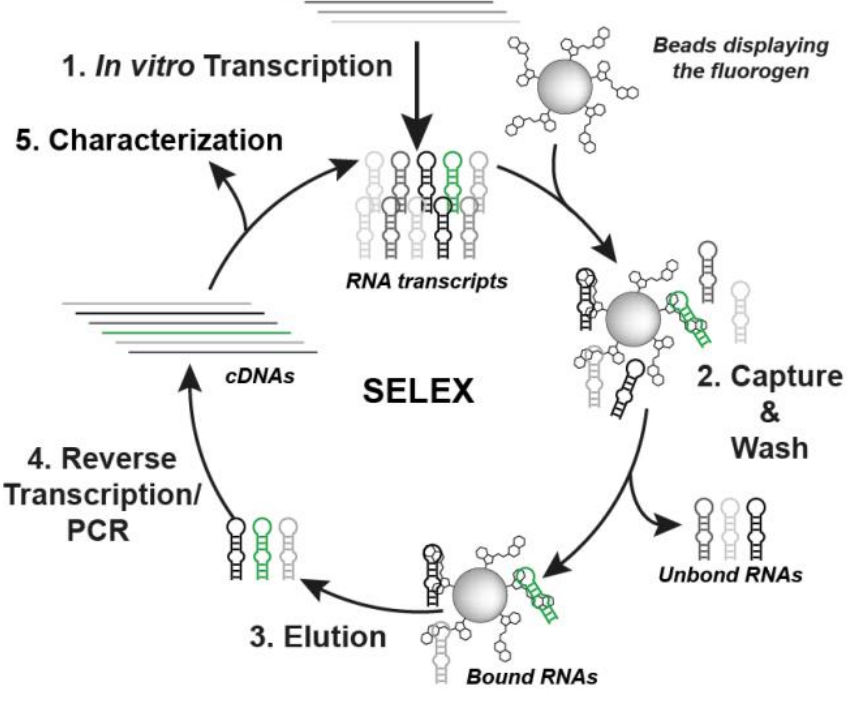

b 1. Bacterial expression or display on beads

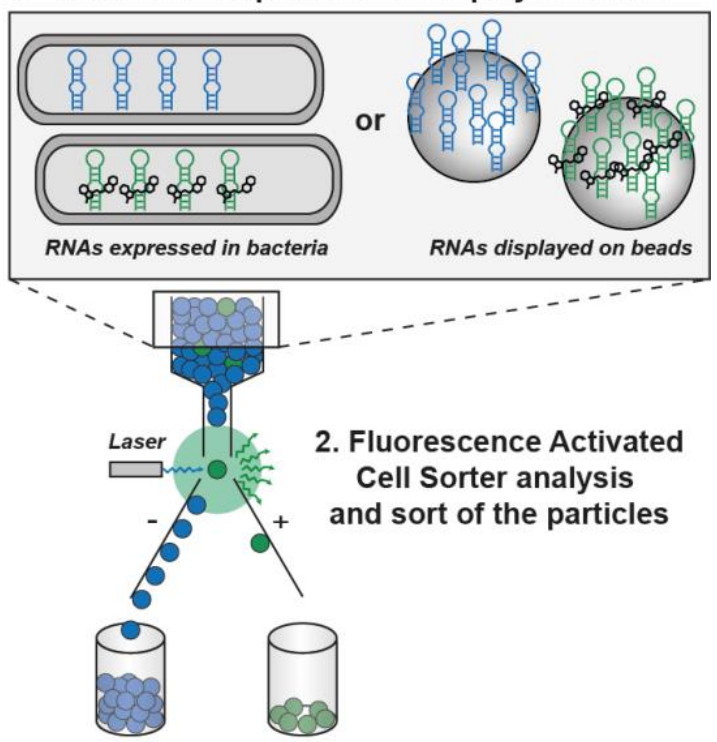

C
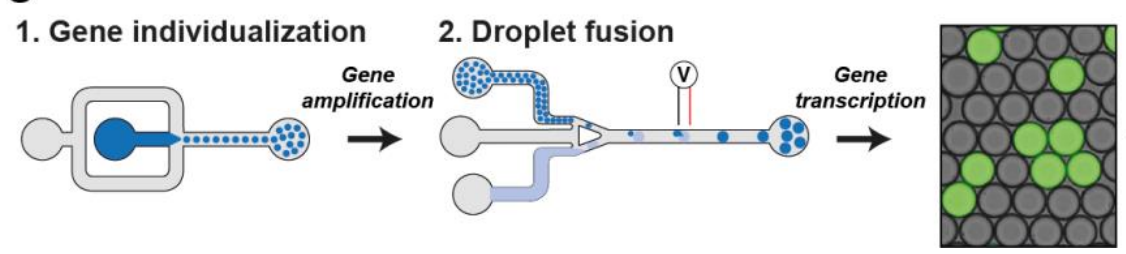

3. Droplet sorting

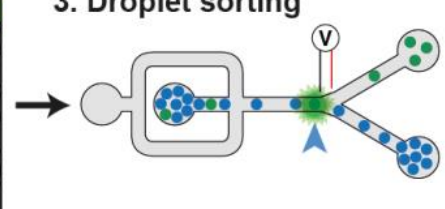

Figure 3. Principal technologies available for selecting light-up RNA aptamers. a. In vitro selection using Systematic Evolution of Ligands by EXponential enrichment (SELEX). Gene libraries are in vitro transcribed into RNAs later challenged to interact with a fluorogen immobilized on beads. Selection pressures are mainly applied to select aptamers able to bind the fluorogen with high affinity. This approach has been used to isolate most of the aptamers listed in table 1. b. Functional screening of aptamers using Fluorescence Activated Cell Sorter (FACS). Gene libraries are expressed in RNAs either in bacteria [35] or at the surface of beads [37]. Upon incubation with the fluorogen, the fluorescence of the particles (bacteria or beads) is analyzed on a FACS and used to sort particles displaying the highest fluorescence and, therefore, contain/display efficient light-up aptamers. c. Functional screening of aptamers using microfluidic-assisted In Vitro Compartmentalization ( $\mu$ IVC). Genes contained in a library are individualized (step 1) together with a PCR amplification mixture in droplets (dark blue) carried by an oil phase (gray). Droplets are collected and thermocycled prior to being reinjected into a droplet fusion device where each DNA-containing droplet (dark blue) is fused to a larger droplet containing an in vitro transcription mixture (light blue) supplemented with fluorogen (step 2). Upon an incubation allowing amplified DNA to be in vitro transcribed, the fluorescence of each droplet is analyzed. To do so, droplets are reinjected into a sorting device in which the fluorescence of each droplet is measured and used to isolate droplets displaying the highest fluorescence and, thus, contain efficient light-up aptamers. This process was used to isolate several aptamers listed in table $1[42,43,56]$. 
a. T01-Biotin/Mango

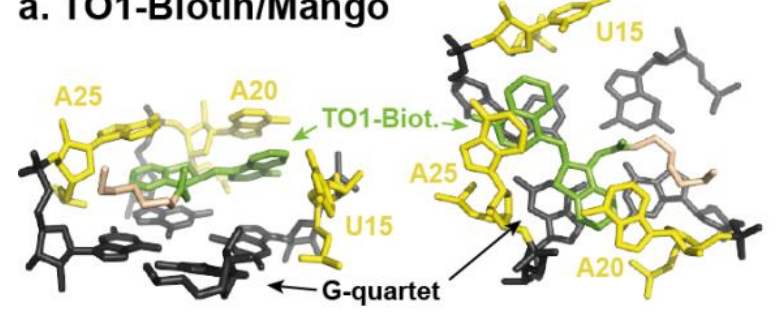

b. T01-Biotin/Mango-II

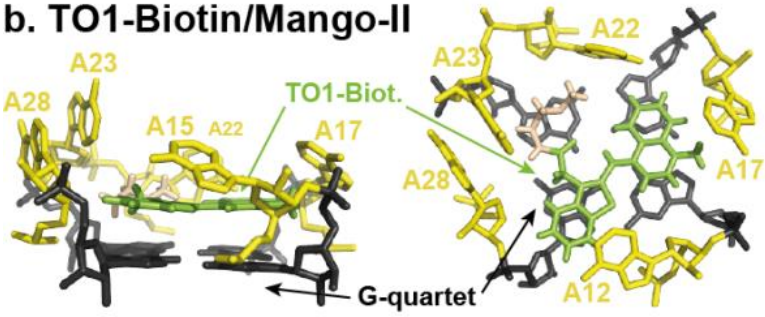

c. T01-Biotin/Mango-III

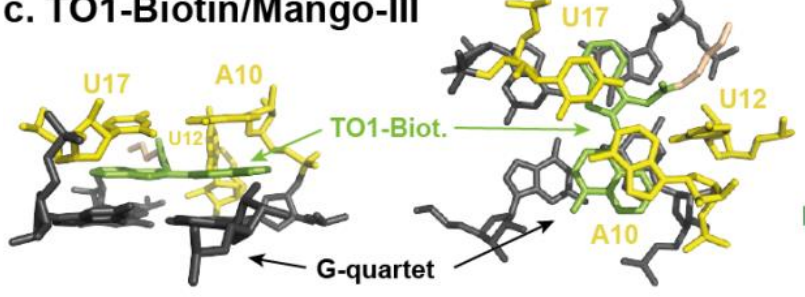

\section{d. OTB-SO3/DIR2s}

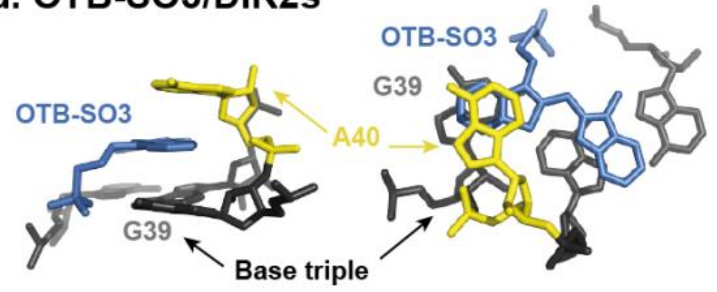

e. Malachite Green/MGA

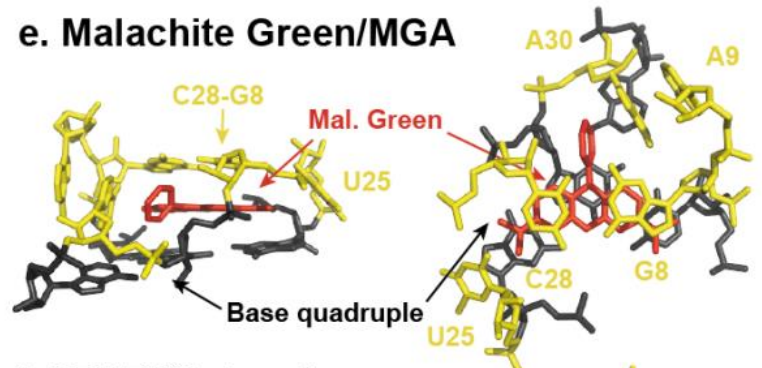

f. DFHBI/Spinach

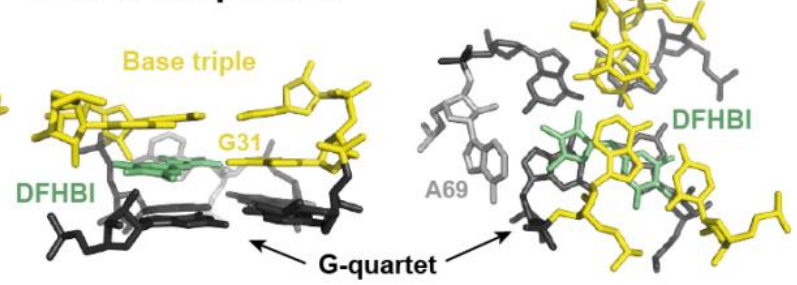

g. DFHO/Corn

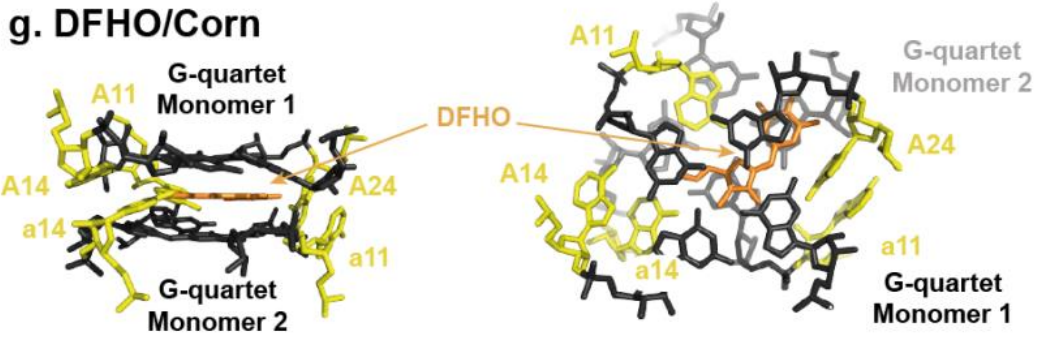

Figure 4. Structural organization of the main fluorogen-binding sites. a. Binding of TO1-Biotin to Mango aptamer. Based on model PDB 5V3F [52]. b. Binding of TO1-Biotin to Mango-II aptamer. Based on model PDB 6C63 [54]. c. Binding of TO1-Biotin to Mango-III aptamer. Based on model PDB 6E8S [55]. d. Binding of OTB-SO3 to DIR2s aptamer. Based on model PDB 6DB8 [47]. e. Binding of Malachite Green to MGA aptamer. Based on model PDB 1F1T [48]. f. Binding of DFHBI to Spinach aptamer. Based on model PDB 4TS2 [50]. g. Binding of DFHO to Corn aptamer. Based on model PDB 5BJO [62]. Nucleotides contributed by Monomer1 (upper case nucleotides) or distinguished from those contributed by Monomer2 (lower case nucleotides). In every model, only the elements in direct contact with the fluorogen are represented. For G-quadruplex-containing RNAs, only the proximal quartet is shown. For each fluorogen/aptamer pair a side (left picture) and top (right picture) view of the complex is represented. Elements are colored as follows: fluorogen-binding platform (G-quartet, base quadruple or base triple) in dark gray, surrounding and capping residues in yellow and fluorogen colored according to their emission wavelength (i.e., OTB-SO3 in bleu, TO1-Biotin and DFHBI in green, DFHO in orange and Malachite Green in red). 

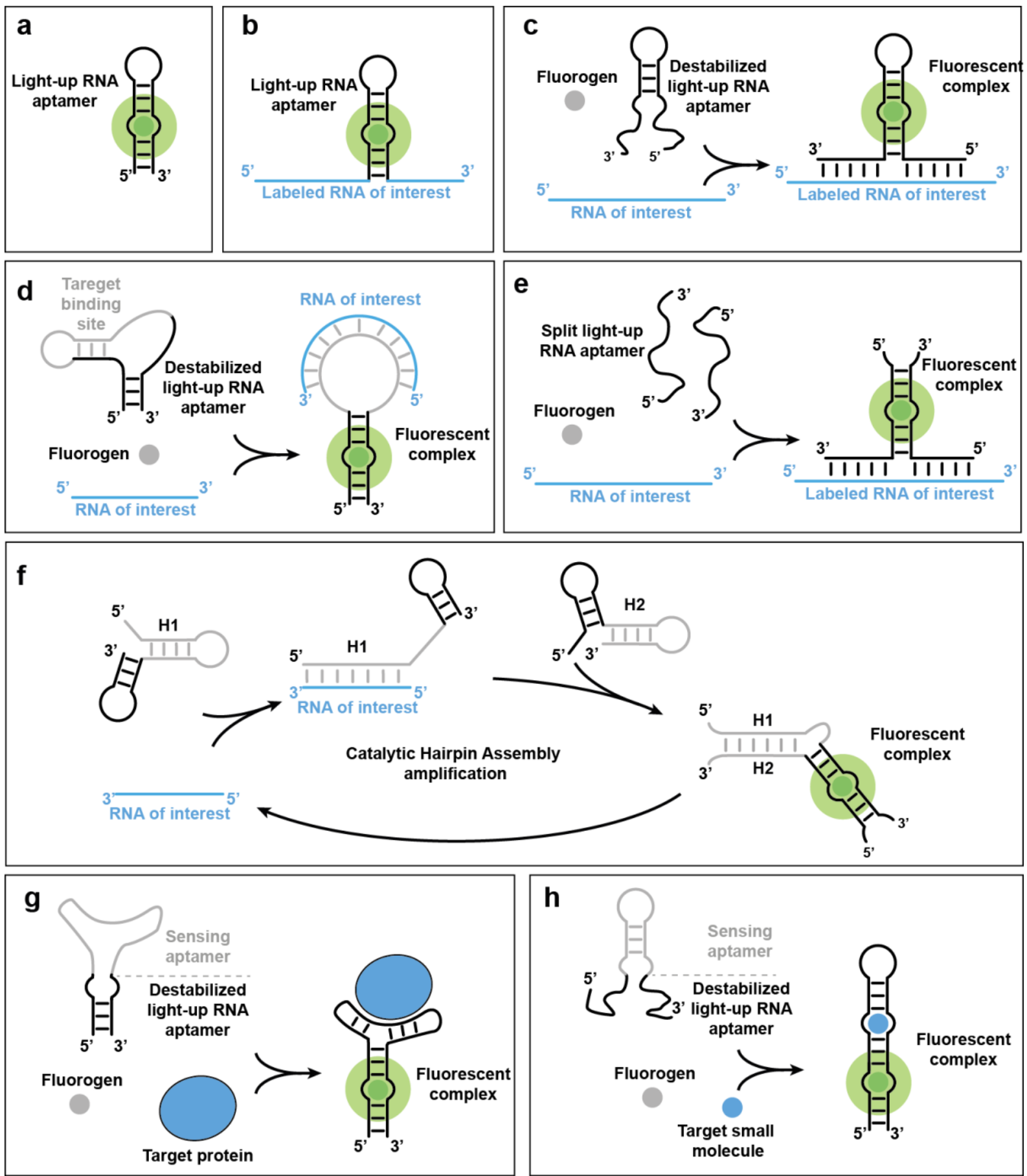

Figure 5. Main labeling/sensing strategies using light-up RNA aptamers. a. Light-up RNA aptamer in complex with its fluorogen. b. Light-up RNA aptamer directly inserted into the RNA of interest. c. RNA-specific probe acting in trans based on transient structural destabilization. d. RNA-specific probe acting in trans based on strand-displacement/structural remodeling. e. RNA-specific probe acting in trans based on a split light-up RNA aptamer. f. RNA-specific probe acting in trans combining stranddisplacement and split light-up aptamers together with Catalytic Hairpin Assemblies amplification circuit (CHARGE technology) [83]. g. Protein sensor using light-up RNA aptamer transiently structurally stabilized as read-out [109]. h. Metabolite sensor using light-up RNA aptamer transiently structurally stabilized as read-out [117]. 